TRANSACTIONS OF THE

AMERICAN MATHEMATICAL SOCIETY

Volume 356, Number 12, Pages 4873-4896

S 0002-9947(04)03516-0

Article electronically published on January 29, 2004

\title{
CHANGE OF RINGS IN DEFORMATION THEORY OF MODULES
}

\author{
RUNAR ILE
}

\begin{abstract}
Given a $B$-module $M$ and any presentation $B=A / J$, the obstruction theory of $M$ as a $B$-module is determined by the usual obstruction class $\mathrm{o}_{A}$ for deforming $M$ as an $A$-module and a new obstruction class $\mathrm{o}_{J}$. These two classes give the tool for constructing two obstruction maps which depend on each other and which characterise the hull of the deformation functor. We obtain relations between the obstruction classes by studying a change of rings spectral sequence and by representing certain classes as elements in the Yoneda complex. Calculation of the deformation functor of $M$ as a $B$-module, including the (generalised) Massey products, is thus possible within any $A$-free 2-presentation of $M$.
\end{abstract}

\section{INTRODUCTION}

In this article we study the following functor of infinitesimal deformations.

Definition 1. Let $A$ be a (commutative) flat $\mathcal{O}$-algebra where $\mathcal{O}$ is a local complete Noetherian ring with $k$ as residue field. Let $\bar{A}=A \otimes_{\mathcal{O}} k$ and let $M$ be an $\bar{A}$-module. Define $\operatorname{Art}_{\mathcal{O}}$ to be the category of local commutative Artinian $\mathcal{O}$-algebras $R$ with residue field $k$ such that the composition $\mathcal{O} \rightarrow R \rightarrow k$ equals the canonical map from $\mathcal{O}$ to its residue field. Morphisms are maps of local $\mathcal{O}$-algebras. The deformation functor of $M$ is a covariant functor

$$
\operatorname{Def}_{M}^{A}: \text { Art }_{\mathcal{O}} \longrightarrow \text { Sets, }
$$

where $\operatorname{Def}_{M}^{A}(R)$ is the set of equivalence classes of deformations of $M$ to $R$. A deformation (or flat lifting) of $M$ to $R$ is an $A_{R}:=A \otimes_{\mathcal{O}} R$-module $M_{R}$, flat as an $R$ module together with an $A_{R}$-linear map $\pi: M_{R} \rightarrow M$ with $\pi \otimes_{R} k: M_{R} \otimes_{R} k \stackrel{\simeq}{\longrightarrow} M$. Two deformations are equivalent if they are isomorphic above $M$. Maps are induced by tensorisation.

Remark 1. One natural choice for $\mathcal{O}$ is as the hull of the deformation functor of $\bar{A}$ as a $k$-algebra, with $A$ the formally versal formal family which in particular is a flat $\mathcal{O}$-algebra with $A \otimes_{\mathcal{O}} k=\bar{A}$. In the case $\mathcal{O}=k$ we have $\bar{A}=A$ and the article might be somewhat easier to read with this assumption.

Received by the editors April 20, 2003.

2000 Mathematics Subject Classification. Primary 13D10, 14B10; Secondary 13D07.

Key words and phrases. Obstruction, Massey product, spectral sequence, mixed characteristic.

This article is based on parts of the author's 2001 Ph.D. Thesis at the Department of Mathematics, University of Oslo. 
More generally, let $F: \operatorname{Art}_{\mathcal{O}} \rightarrow$ Sets be a covariant functor with $F(k)$ a oneelement set. M. Schlessinger [19] formulated a sufficient and necessary set of criteria for the existence of a complete local ring $H$, called a (pro-representing) hull, and a formally versal formal family $\left\{M_{n}\right\}_{n=1}^{\infty}$, a projective system with $M_{n} \in$ $F\left(H / \mathfrak{n} \mathfrak{m}_{H}^{n-1}\right)$, where $\mathfrak{n}=\mathfrak{m}_{H}^{2}+\mathfrak{m}_{\mathcal{O}} H$ such that the induced map

$$
\rho: \operatorname{Hom}_{\mathcal{O}-\text { alg. } / k}^{\text {cont. }}(H,-) \rightarrow F
$$

is formally smooth and an isomorphism on the relative Zariski tangent space. $F$ is called pro-representable if $\rho$ is an isomorphism. Most deformation functors have hulls if the relative Zariski tangent space of $F$ is finite dimensional.

Example 1. Let $\rho: \Pi \rightarrow \mathrm{Gl}_{n}(k)$ be a continuous representation of a profinite group $\Pi$ satisfying a $p$-finiteness condition, where $p$ is the characteristic of the finite field $k$. Define the deformation functor $\operatorname{Def}_{\rho}: \operatorname{Art}_{\mathcal{O}} \rightarrow$ Sets as equivalence classes of liftings $\widetilde{\rho}: \Pi \rightarrow \mathrm{Gl}_{n}(R)$ of $\rho$. Here $\mathcal{O}$ is a "coefficient ring" with residue field $k$, typically $\mathcal{O}=W(k)$, the Witt ring of $k$. If $\Pi$ is the Galois group of a number field, one obtains what B. Mazur has termed deformation theory of Galois representations; cf. [17]. If $A=\mathcal{O}[[\Pi]]$ and $M=k^{n}$ with the $\bar{A}=k[[\Pi]]$-module structure induced from $\rho$, this deformation functor is canonically isomorphic to the one in Definition 1 (we have to allow for non-commutative algebras $A$ ). By applying the Schlessinger criteria, Mazur proved that Def ${ }_{\rho}$ in general has a hull, and is pro-representable if $\rho$ is absolutely irreducible; see [16].

Example 2. If $\mathcal{O}=k$ is an algebraically closed field of any characteristic and $M$ is a finitely generated $A$-module (and $A$ is an algebraic $k$-algebra, i.e. the Henselisation of a finitely generated $k$-algebra at a maximal ideal), locally free on the complement of the closed point, there exist algebraic versal deformations of $M[5,22$. A. Ishii [12] has constructed a filtration of the versal base spaces (with reduced structure) of all reflexive modules (including the decomposable ones) on rational surface singularities and has determined the local deformation relation of the reflexive modules on the rational double points. These mini-versal base spaces are far from being (locally) "coarse" moduli spaces. In particular, the deformation functors of these reflexive modules restricted to Artinian rings are not pro-representable. Indeed there is only a finite set of isomorphism classes of reflexive modules of fixed rank on a quotient surface singularity, yet the singular versal base has complicated geometry.

Schlessinger did not provide any effective construction of the hull. The only known general method to compute $H$ given $M$ is via a natural obstruction class.

Definition 2. A small lifting situation is a surjective map $\pi: R \rightarrow S$ in $\operatorname{Art}_{\mathcal{O}}$, where $\operatorname{ker} \pi$ is contained in the socle of $R$, i.e. $\mathfrak{m}_{R} \cdot \operatorname{ker} \pi=0$, and a deformation $M_{S}$ of $M$ to $S$.

The obstruction class is then an element $\mathrm{o}_{*}=\mathrm{o}_{*}\left(\pi, M_{S}\right) \in \mathrm{H}^{2} \otimes \operatorname{ker} \pi$, where $\mathrm{H}^{2}$ is the second cohomology group of the object $M$. If $F=\operatorname{Def}_{M}^{A}$, then $\mathrm{o}_{A}=\mathrm{o}_{A}\left(\pi, M_{S}\right)$ and $\mathrm{H}^{2}=\operatorname{Ext}_{\frac{A}{A}}(M, M)$. The obstruction class is natural with respect to morphisms of the lifting situation. There exists a lifting of $M_{S}$ to $R$ (or a prolongation of the deformation $M_{S}$ to the "thicker" Artinian neighbourhood $\operatorname{Spec} R$ ) if and only if this obstruction class is zero. The obstruction class has been constructed for many deformation functors, e.g. [10] 11, 14]. For axiomatic approaches see [1, 6, 8]. 
If $F$ has a hull, there is a universal element $M_{1} \in F\left(H_{1}\right)$, where $H_{1}=k\left[\mathrm{H}^{1^{*}}\right]=$ $k \oplus \mathrm{H}^{1^{*}}$ and $\mathrm{H}^{1}$ is the relative Zariski tangent space; $\mathrm{H}^{1} \cong F(k[\varepsilon]$ ) (naturally a $k$-vector space). In the case $F=\operatorname{Def}_{M}^{A}, \mathrm{H}^{1}=\operatorname{Ext} \frac{1}{A}(M, M)$ and $M_{1}$ is given by the universal extension

$$
M_{1}: \quad 0 \longrightarrow M \otimes_{k} \operatorname{Ext} \frac{1}{A}(M, M)^{*} \longrightarrow M_{1} \stackrel{\pi_{1}}{\longrightarrow} M \longrightarrow 0 .
$$

The construction of $H$ then proceeds through successive "prolongations" of $M_{1}$ to thicker Artinian $\mathcal{O}$-algebras through small lifting situations, at each step calculating the obstruction. If this is done correctly, one obtains power series in $T^{1}$, contained in $\mathfrak{m}_{T^{1}}^{2}+\mathfrak{m}_{\mathcal{O}} T^{1}$, one (possibly " 0 ") for each generator in $T^{2}$ of the relative cotangent space, where $T^{i}$ is the completion of the free $\mathcal{O}$-algebra which has $\mathrm{H}^{i}$ as relative Zariski tangent space for $i=1,2$. This defines an obstruction map $\mathrm{o}^{*}: T^{2} \rightarrow T^{1}$, which is naturally compatible with the obstruction class $\mathrm{O}_{*}$ (see Definition [5), such that $H=T^{1} \hat{\otimes}_{T^{2}} \mathcal{O}$.

The existence of an obstruction map is provided by O. A. Laudal rather abstractly for a deformation functor of a small category of algebras in [14. Thm. 4.2.4] (see also V. P. Palamodov [18. Thm. 5.6] (without proof) for compact analytic manifolds) and for $\operatorname{Def}_{M}^{A}$ and $\mathcal{O}=k$ with explicit Yoneda-representations of the generalised Massey products in [15]. For an axiomatic existence theorem, see [8. Thm. 2.3.10]. It shows that the existence of a natural obstruction class, together with a natural action of the tangent space on the set of liftings in a small lifting situation, implies the existence of an obstruction map for $F$. Once we have an obstruction map, the general Krull dimension estimate

$$
\operatorname{dim}_{k} \mathrm{H}^{1} \geqslant \operatorname{dim}_{\text {Krull }} H-\operatorname{dim}_{\text {Krull }} \mathcal{O} \geqslant \operatorname{dim}_{k} \mathrm{H}^{1}-\operatorname{dim}_{k} \mathrm{H}^{2}
$$

follows. (See also [13].)

In practice it is difficult to give non-trivial results about the obstruction map; the usual application is some variation of $\mathrm{H}^{2}=0 \Rightarrow H$ is smooth. In fact, very few classes of examples of deformation functors have been given for which anything beyond the general Krull dimension estimate is known. By studying modules, one can at least calculate examples as there exists an effective obstruction algorithm. In the present paper we provide a refinement of the obstruction map for modules which has both theoretical and computational consequences. For an application of these ideas, see [9].

Let $B$ be a flat $\mathcal{O}$-algebra which is a quotient of $A$, let $J=\operatorname{ker}(A \rightarrow B)$ and assume $M$ is a $\bar{B}=B \otimes_{\mathcal{O}} k$-module as $\bar{A}$-module, i.e. that $\bar{J}=J \otimes_{\mathcal{O}} k \subseteq \operatorname{Ann}_{\bar{A}}(M)$. Suppose we want to study the deformation functor of $B$-modules $\operatorname{Def}_{M}^{B}$. The $\bar{B}$ cohomology of $M$ may be complicated while $A$ can be chosen as a simpler ring. There is a natural injective map $\operatorname{Def}_{M}^{B} \rightarrow \operatorname{Def}_{M}^{A}$ and the ideal $J$ acts on an $A$ deformation $M_{R}$ of $M$ to $R$ through the $A_{R}$-action. Let $\operatorname{Def}_{M}^{(A, J)} \subseteq \operatorname{Def}_{M}^{A}$ be the sub-functor of $A$-deformations annihilated by $J$.

Lemma 1. Let $A$ and $B$ be flat $\mathcal{O}$-algebras and $M a \bar{B}=B \otimes_{\mathcal{O}} k$-module. Let $J$ be an ideal in $A$ and assume $B=A / J$. Then

$$
\operatorname{Def}_{M}^{B} \cong \operatorname{Def}_{M}^{(A, J)}
$$

The main idea in this paper emerges from Lemma[1; Lift $M$ as an $A$-module with trivial $J$-action and only use $\bar{A}$-cohomology to characterise the tangent space and the obstructions. In Theorem 1 we give a new obstruction class $\mathrm{o}_{J}$ which exists (in a 
small lifting situation) if the obstruction $\mathrm{o}_{A}$ for lifting $M$ as $A$-module is zero, such that $\mathrm{o}_{J}=0$ if and only if there exists an $A$-lifting with trivial $J$-action. In fact $\mathrm{o}_{J}$ will sit in the cokernel of a natural map $\partial_{\bar{J}}: \operatorname{Ext} \frac{1}{A}(M, M) \rightarrow \operatorname{Hom}_{\bar{A}}\left(\bar{J}, \operatorname{End}_{\bar{A}}(M)\right)$. Moreover, the kernel of this map is the tangent space of $\operatorname{Def}_{M}^{(A, J)}$.

With two natural obstruction classes we construct two obstruction maps $\left(\mathrm{o}^{A}, \mathrm{o}^{J}\right)$, in Theorem [2, which are compatible with the obstructions (see Definition [5). The obstruction maps are defined if the cohomology $k$-vector spaces are of countable dimension, as in [14]. Remark how these maps depend on each other. In particular, it is not true that $\mathrm{o}^{A}$ in the pair $\left(\mathrm{o}^{A}, \mathrm{o}^{J}\right)$ is induced by $\mathrm{o}^{A}$ for $\operatorname{Def}_{M}^{A}$ as we clearly see in Example 5. This example also shows that much of the obstruction space is not necessarily "hit" by obstructions (at least as long as we do not deform over noncommutative Artinian algebras). Theorem 3 compares $\left(\mathrm{o}^{A}, \mathrm{o}^{J}\right)$ with the traditional $\mathrm{O}^{B}$ and is based on the relations of the various obstruction classes which are found by investigating maps in a change of rings spectral sequence, which is undertaken in Section 4 and Section 5 . In particular; Theorem 4 ties several of our obstruction classes together by a cup product with the obstruction class for lifting $M$ non-flat to $\bar{A} / \bar{J}^{2}$. Finally, in Section [6] we give three obstruction calculations. In Example [6] and 7]we find obstructions in mixed characteristic. In Example 8 the obstruction ideal is given as a regular sequence (at least in an infinite set of cases) with two elements while $\operatorname{dim}_{k} \operatorname{Ext}_{B}^{2}(M, M)=4$. Indeed it might be a good idea to take a look at the examples in Section 6 before reading Section 275 .

For actual calculation of the obstruction power series, one can lift a free resolution of the module; see [15]. The universal deformation to the relative Zariski tangent space of the deformation functor is given by perturbing the differentials in the resolution with Yoneda-representations for a $k$-basis of $\operatorname{Ext}^{1}(M, M)$. The quadratic obstruction is given in terms of cup products and the higher degree obstruction as generalised Massey products which are represented as composition products in the Yoneda algebra. It is therefore not sufficient for our purposes to work in the derived category, and our results describing maps in the change of rings spectral sequence and the comparison of obstruction classes is done by giving explicit representations in the appropriate Yoneda algebra of a free complex. Our result enables the obstruction calculus to be performed entirely within a (truncated) Yoneda complex of an $A$-free resolution of the $B$-module $M$. A formal proof of this (in the case $\mathcal{O}=k$ ) is given in 8, Thm. 3.3.2]; see also Example6 8 .

For explicit non-trivial calculations of obstructions (given by cup products) for the Hilbert functor of space curves, see [23, 7]. A. Siqveland gave the local equations for the compactified Jacobian of the $\mathbf{E}_{\mathbf{6}}$ curve singularity and found the degeneracy diagram of the rank 1 torsion free modules in [20] by calculating the obstruction maps. The Massey product algorithms are given in [21]. Similar ideas have recently been used by I. C. Borge and O. A. Laudal 3] to solve the modular isomorphism problem for $p$-groups with $\mathbb{F}_{p}$-coefficients. See also [2].

\section{The $J$-OBSTRUCTION CLASS}

In this section we construct 3 obstruction classes for lifting a module in a relative lifting situation.

Let $A \rightarrow B$ be any surjective ring homomorphism and let $M$ and $N$ be $A$ modules with $A$-free resolutions $F$ and $F^{\prime}$, respectively. The corresponding Yoneda complex is the differential graded module $\operatorname{Hom}_{A}^{*}\left(F, F^{\prime}\right)$, where $\operatorname{Hom}_{A}^{n}\left(F, F^{\prime}\right)=$ 
$\operatorname{Hom}_{A}\left(F[n], F^{\prime}\right)$ with differential $\partial$ induced from the differentials $d$ and $d^{\prime}$ on $F$ and $F^{\prime}$, respectively. If $\xi \in \operatorname{Hom}_{A}^{n}\left(F, F^{\prime}\right)$, i.e. $\xi=\left(\xi_{i}\right)_{i}$ with $\xi_{i}: F_{i+n} \rightarrow F_{i}^{\prime}$, then $\partial \xi=d^{\prime} \xi-(-1)^{n} \xi d=\left(d_{i+1}^{\prime} \xi_{i+1}-(-1)^{n} \xi_{i} d_{i+1+n}\right)_{i}$. We have $\mathrm{H}^{n} \operatorname{Hom}_{A}^{*}\left(F, F^{\prime}\right) \cong$ $\operatorname{Ext}_{A}^{n}(M, N)$. Our first objective is to define a lifting of a Tor-action on $\operatorname{Ext}_{A}^{*}(M, N)$ to the Yoneda complex, which will enable us to study the $J$-action on the $A$ deformations of $M$. Assume that $M$ and $N$ are $B$-modules as $A$-modules and let $E$ be an $A$-free resolution of the $A$-module $B$.

Let $m: E \otimes_{A} F \rightarrow F$ and $m^{\prime}: E \otimes_{A} F^{\prime} \rightarrow F^{\prime}$ lift $B \otimes_{A} M \cong M$ and $B \otimes_{A} N \cong N$, respectively, and see that for $e \in E, m, m^{\prime}$ give $m(e) \in \operatorname{End}_{A}(F)$, respectively, $m^{\prime}(e) \in \operatorname{End}_{A}\left(F^{\prime}\right)$. Define

$$
\partial_{A / B}: E \longrightarrow \operatorname{End}_{A}^{*}\left(\operatorname{Hom}_{A}^{*}\left(F, F^{\prime}\right)\right)
$$

by $\partial_{A / B}(e)(\varphi)=m^{\prime}(e) \varphi-(-1)^{|\varphi||e|} \varphi m(e)$, where $\varphi \in \operatorname{Hom}_{A}^{*}\left(F, F^{\prime}\right)$. Clearly $\partial_{A / B}$ depends on the choices made.

Proposition 1. $\partial_{A / B}$ induces a canonical map of graded B-modules

$$
\operatorname{Tor}_{*}^{A}(B, B) \longrightarrow \operatorname{End}_{A}^{*}\left(\operatorname{Ext}_{A}^{*}(M, N)\right)
$$

making $\operatorname{Ext}_{A}^{*}(M, N)$ a $\operatorname{Tor}_{*}^{A}(B, B)$-module. In the case $M=N, \operatorname{Ext}_{A}^{*}(M, M)$ is an algebra-module and $\operatorname{Tor}_{p}^{A}(B, B)$ acts as degree $p$-derivations. The map is natural in the sequence $(A \rightarrow B, M, N)$.

Proof. One calculates

$$
\partial_{A / B}(e)(\partial \varphi)= \pm \partial\left(\partial_{A / B}(e)(\varphi)\right)
$$

hence we get induced a $\partial_{A / B}: E \longrightarrow \operatorname{End}_{A}^{*}\left(\operatorname{Ext}_{A}^{*}(M, N)\right)$. It factorises via $B \otimes_{A} E$ and one again calculates

$$
\partial_{A / B}(d \bar{e})(\varphi)= \pm \partial\left(\partial_{A / B}(\bar{e})(\varphi)\right)
$$

if $\partial \varphi=0$. We get a map

$$
\mathrm{H}\left(B \otimes_{A} E\right) \longrightarrow \operatorname{End}_{A}^{*}\left(\operatorname{Ext}_{A}^{*}(M, N)\right)
$$

which is independent of the choices made.

Definition 3. Let $J=\operatorname{ker}(A \rightarrow B)$ and define

$$
\partial_{J}: \operatorname{Ext}_{A}^{1}(M, N) \longrightarrow \operatorname{Hom}_{A}\left(J, \operatorname{Hom}_{A}(M, N)\right)
$$

to be the adjoint of $\partial_{A / B}$ restricted to $J$ through $\operatorname{Tor}_{1}^{A}(B, B) \cong J / J^{2}$. In terms of Yoneda representations (see notation before Proposition 1), if $E_{1}=A^{r} \rightarrow J=$ $\left(f_{1}, \ldots, f_{r}\right)$ and $m_{1}: E_{1} \otimes_{A} F_{0} \rightarrow F_{1}$ is $A$-linear such that $d_{1} m_{1}$ induces the multiplication with $\left(f_{1}, \ldots, f_{r}\right)$ on $F_{0}, J \otimes_{A} F_{0} \rightarrow F_{0}$, then $\partial_{J}([\xi])$ is defined as the adjoint of the class in $\operatorname{Hom}_{A}\left(J \otimes_{A} M, N\right)$ induced by $\xi_{0} m_{1}: E_{1} \otimes_{A} F_{0} \rightarrow F_{0}^{\prime}$, where $\xi_{0}: F_{1} \rightarrow F_{0}^{\prime}$ represents $[\xi]$.

For convenience we will also use the $\partial_{J}$-notation in adjoint situations, as in the next theorem.

We are now in the position to formulate necessary and sufficient conditions for the existence of deformations of an $A$-module with trivial $J$-action, i.e. a $B$-module, in a small lifting situation. The standard result here, as given in [15], is to produce a class $\mathrm{O}_{B}$ in the $B$-cohomology of the situation, for modules that would be $\mathrm{Ext}_{B}^{2}$, which vanish if and only if there is a lifting. We will instead produce two classes (actually three), the $\mathrm{o}_{A}$ in $\operatorname{Ext}_{A}^{2}$ which is the old obstruction for lifting $A$-modules, 
and if $\mathrm{o}_{A}=0$, a new class $\mathrm{o}_{J}$, also given by $A$-cohomology such that $\mathrm{o}_{J}=0$ if and only if there exists an $A$-lifting with trivial $J$-action. These two classes will enable us to characterise the hull of $\operatorname{Def}_{M}^{B}$ by two obstruction maps (see Theorem 21) in the same way as $\mathrm{O}_{B}$ gives the tool for constructing the obstruction map in [15].

We formulate the result in the following relative lifting situation: Suppose $A$ and $B$ are flat $\mathcal{O}$-algebras, where $\mathcal{O}$ is a commutative ring. Let $A \rightarrow B$ and $\pi: R \rightarrow S$ be surjective maps of $\mathcal{O}$-algebras with kernels the ideals $J$ and $I$, respectively. Let $M$ be a $B_{S}=B \otimes_{\mathcal{O}} S$-module (similarly $J_{S}=J \otimes_{\mathcal{O}} S$ etc.). Assume $I^{2}=0$.

Definition 4. A lifting of $M$ to $B_{R}$ is a $B_{R}$-module $M_{R}$ and a $B_{R}$-linear map $\pi: M_{R} \rightarrow M$ with $\pi \otimes S: M_{R} \otimes_{R} S \stackrel{\simeq}{\longrightarrow} M$, such that $\operatorname{Tor}_{1}^{R}\left(M_{R}, S\right)=0$.

Theorem 1. In a relative lifting situation as above we have:

i) There exists a class $\mathrm{o}_{A_{S}}=\mathrm{o}_{A_{S}}(\pi, M) \in \operatorname{Ext}_{A_{S}}^{2}\left(M, M \otimes_{S} I\right)$ such that $\mathrm{o}_{A_{S}}=0$ if and only if there exists a lifting of $M$ to $A_{R}$.

ii) Given a lifting $M_{R}$ of $M$ to $A_{R}$, there exists a class

$$
\mathrm{o}\left(J_{S}\right) \in \operatorname{Hom}_{A_{S}}\left(J_{S} \otimes_{A_{S}} M, M \otimes_{S} I\right)
$$

such that $\mathrm{o}\left(J_{S}\right)=0$ if and only if $M_{R}$ is a $B_{R}$-module as an $A_{R}$-module.

iii) If $\mathrm{o}_{A_{S}}=0$ there exists a class $\mathrm{o}_{J_{S}}=\mathrm{o}_{J_{S}}(\pi, M) \in$ coker $\partial_{J_{S}}$, where

$$
\partial_{J_{S}}: \operatorname{Ext}_{A_{S}}^{1}\left(M, M \otimes_{S} I\right) \longrightarrow \operatorname{Hom}_{A_{S}}\left(J_{S} \otimes_{A_{S}} M, M \otimes_{S} I\right)
$$

is as given in Definition 3, such that $\mathrm{o}_{J_{S}}=0$ if and only if there is a lifting of $M$ to $B_{R}$.

iv) Assume $\mathrm{o}_{A_{S}}=0=\mathrm{O}_{J_{S}}$. Then there is a transitive and effective action of ker $\partial_{J_{S}}$ on the set of isomorphism classes of liftings of $M$ to $B_{R}$ over $M$.

Finally, all classes and the action are natural for flat maps of $A \rightarrow B$ and of $R \rightarrow S$ and the induced modules. If $M$ is $S$-flat, the naturality follows for all maps of $R \rightarrow S$.

Remark 2. In the case $\mathcal{O}=k=A=B$ (and $J=0$ ) one recovers the standard result and our construction of $o_{S}$ is as in [15].

Remark 3. By Lemma $4 \mathrm{o}_{A_{S}}$ is in the image of the natural map $\operatorname{Ext}_{B_{S}}^{2} \rightarrow \operatorname{Ext}_{A_{S}}^{2}$.

Proof. Since we are mainly interested in the deformation case, we give a proof under the additional assumption of either $M$ or $I$ being $S$-flat. The complex we use lends itself best to these cases. At the end we comment on the general situation.

i) Let $F=(F, d)$ be an $A_{S}$-free resolution of $M$. By the freeness we can lift the differential to a map $\tilde{d}$ of the graded $A_{R}$-free module $\tilde{F}$ which in each degree has the same rank as $F$, thus $\tilde{d} \otimes_{R} S=d$. If $\tilde{F}=(\tilde{F}, \tilde{d})$ were a complex, it would be a resolution of a lifting of $M$ to $A_{R}$. Tensoring $\tilde{F}$ with the short exact sequence $0 \rightarrow$ $I \rightarrow R \stackrel{\pi}{\longrightarrow} S \rightarrow 0$ of $R$-modules gives an exact sequence $0 \rightarrow F \otimes_{S} I \rightarrow \tilde{F} \stackrel{\pi}{\longrightarrow} F \rightarrow 0$ of graded modules since $A$ is flat as $\mathcal{O}$-module. It follows that $(\tilde{d})^{2}$ is induced by a map $\rho \in Z^{2} \operatorname{Hom}_{A_{S}}\left(F, F \otimes_{S} I\right)$, i.e. a 2-cocycle in the Yoneda complex. Define

$$
\mathrm{o}_{A_{S}}=\mathrm{o}_{A_{S}}(\pi, M):=[\rho] \in \operatorname{Ext}_{A_{S}}^{2}\left(M, M \otimes_{S} I\right) \text {. }
$$

This element is independent of the resolution $F$ and the choice of lifting $(\tilde{F}, \tilde{d})$ and is the obstruction for lifting $M$ along $\pi$ : If $\mathrm{o}_{A_{S}}=0$, there is a $\tau \in \operatorname{Hom}_{A_{S}}^{1}\left(F, F \otimes_{S} I\right)$ this is the place where we use the additional hypothesis - with $\partial \tau=\rho$. We perturb $\tilde{d}$ by $\tau$ and get a differential $d_{R}=\tilde{d}-\tau \pi$. Hence $F_{R}=\left(\tilde{F}, d_{R}\right)$ is a complex which 
is an extension of resolutions (by the additional hypothesis again), thus itself is a resolution of $M_{R}:=\mathrm{H}_{0}\left(F_{R}\right)$. Clearly $M_{R} \otimes_{R} S \cong M$ and $\operatorname{Tor}_{1}^{R}\left(M_{R}, S\right)=\mathrm{H}_{1}(F)=$ 0 , in fact $M_{R}$ is $R$-flat if $M$ is $S$-flat. If there is a lifting $M_{R}$ of $M, \mathrm{o}_{A_{S}}=0$ by the independence of the choices we claimed above.

ii) To find the obstruction for $M_{R}$ to be a $B_{R}$-module as $A_{R}$-module, we lift the canonical isomorphism $m_{0}: A_{S} \otimes_{A_{S}} F \rightarrow F$ to a map of complexes $m: E_{S} \otimes_{A_{S}} F \rightarrow$ $F$, where $\ldots \rightarrow E_{2} \rightarrow E_{1} \rightarrow J$ gives an $A$-free resolution of $J$ which, together with $J \subset A=E_{0}$, gives an $A$-free resolution $E$ of $B$, and $E_{S}:=E \otimes_{\mathcal{O}} S$ gives an $A_{S}$-free resolution of $B_{S}$. The lifting $m$ exists since $M$ is a $B_{S}$-module as in Proposition [1] i.e. since $J_{S}$ 's action on $F$ is homotopically trivial. Choose an $\tilde{m}: \quad E_{R} \otimes_{A_{R}} F_{R} \rightarrow F_{R}$ with $\tilde{m} \otimes_{R} S=m$ and with $\tilde{m}_{\mid\left(E_{R}\right)_{0} \otimes F_{R}}$ the canonical isomorphism $A_{R} \otimes_{A_{R}} F_{R} \cong F_{R}$. Then we can view $\tilde{m}$ as an attempt to kill the action of $J_{R}$ on $F_{R}$. We find $\partial(\tilde{m})=d\left(F_{R}\right) \circ \tilde{m}-\tilde{m} \circ d\left(E \otimes F_{R}\right)$ to be induced by a $\rho \in Z^{0} \operatorname{Hom}_{A_{S}}\left(E_{\geqslant 1} \otimes_{A} F, F \otimes_{S} I\right)$, where $E_{\geqslant 1}=\left[\ldots \rightarrow E_{2} \rightarrow E_{1}\right][+1]$. Define

$$
\mathrm{o}\left(J_{S}\right):=[\rho] \in \mathrm{H}^{0} \operatorname{Hom}_{A_{S}}\left(E_{\geqslant 1} \otimes_{A} F, F \otimes_{S} I\right) \cong \operatorname{Hom}_{A_{S}}\left(J_{S} \otimes_{A_{S}} M, M \otimes_{S} I\right) .
$$

This class only depends on the lifting $M_{R}$ and is the obstruction for $M_{R}$ to be a $B_{R}$-module as $A_{R}$-module. If o $\left(J_{S}\right)=0$, there is a $\tau \in \operatorname{Hom}_{A_{S}}^{-1}\left(E_{\geqslant 1} \otimes_{A} F, F \otimes_{S} I\right)$ with $\partial(\tau)=\rho$. Perturbing $\tilde{m}$ with $\tau$ gives $m_{R}=\tilde{m}-\tau \pi$ with $\partial\left(m_{R}\right)=0$.

iii) The $\mathrm{o}(J)$ only checks our specific choice of lifting $M_{R}$ given by $d_{R}$; other choices of $M_{R}$ could be better. To obtain other $A_{R}$-liftings we perturb $d_{R}$ by $\xi \in Z^{1} \operatorname{Hom}_{A_{S}}\left(F, F \otimes_{S} I\right)$ to $d_{R}^{\prime}=d_{R}+\xi \pi$. This gives a new differential $\partial^{\prime}$ and

$$
\begin{aligned}
\partial^{\prime}(\tilde{m}) & =\left(d_{R}+\xi \pi\right) \tilde{m}-\tilde{m}\left(d_{E \otimes F_{R}^{\prime}}\right) \\
& =d_{R} \tilde{m}-(-1)^{|E|} \tilde{m}\left(1 \otimes d_{R}\right)-\tilde{m}\left(d_{E} \otimes 1\right)+\xi \pi \tilde{m}-(-1)^{|E|} \tilde{m}(1 \otimes \xi \pi) \\
& =\left(\rho+\partial_{A_{S} / B_{S}}(\xi)\right) \pi,
\end{aligned}
$$

where $\partial_{A_{S} / B_{S}}: \operatorname{Hom}_{A_{S}}\left(F, F \otimes_{S} I\right) \longrightarrow \operatorname{Hom}_{A_{S}}\left(E \otimes_{A} F, F \otimes_{S} I\right)$ up to adjointness is the one in Proposition 1. Define the class

$$
\begin{aligned}
\mathrm{o}_{J_{S}}=\mathrm{o}_{J_{S}}(\pi, M):= & {\left[\mathrm{o}\left(J_{S}\right)\right] \in \operatorname{coker} \partial_{J_{S}} } \\
& =\operatorname{coker}\left(\operatorname{Ext}_{A_{S}}^{1}\left(M, M \otimes_{S} I\right) \rightarrow \operatorname{Hom}_{A_{S}}\left(J_{S} \otimes_{A_{S}} M, M \otimes_{S} I\right)\right) .
\end{aligned}
$$

It depends only on $M$ and $\pi$, and is the obstruction for lifting $M$ to $B_{R}$ if there exists a lifting of $M$ to $A_{R}$. If $\partial^{\prime}(\tilde{m})=\partial(\tau) \pi$, with $\tau \in \operatorname{Hom}_{A_{S}}^{0}\left(F, F \otimes_{S} I\right)$, we can perturb $\tilde{m}$ to $\tilde{m}^{\prime}=\tilde{m}-\tau \pi$ and $\partial^{\prime}\left(\tilde{m}^{\prime}\right)=\left(\rho+\partial_{A_{S} / B_{S}}(\xi)-\partial \tau\right) \pi=0$ so $\tilde{m}^{\prime}$ gives a homotopy to zero for the action of $J_{R}$ on $F_{R}^{\prime}$, i.e. $M_{R}^{\prime}$ is a $B_{R}$-module as $A_{R}$-module.

iv) It also follows that any $\xi^{\prime} \in Z^{1} \operatorname{Hom}_{A_{S}}\left(F, F \otimes_{S} I\right)$ with $\partial_{A_{S} / B_{S}}\left(\xi^{\prime}\right)=0$ gives another lifting to $B_{R}$ by $d_{R}^{\prime \prime}=d_{R}^{\prime}+\xi^{\prime} \pi$ and that the difference $d_{R}^{\prime}-d_{R}^{\prime \prime}$ of two liftings to $B_{R}$ gives an element in ker $\partial_{A_{S} / B_{S}}$. They are isomorphic if and only if this element is zero in $\operatorname{Ext}_{A_{S}}^{1}\left(M, M \otimes_{S} I\right)$.

For the general case, the main difference is that $F \otimes_{S} I$ is not necessarily a resolution, and a resolution $F_{R}$ of $M_{R}$ will give $\mathrm{H}_{*}\left(F_{R} \otimes_{R} S\right) \cong \operatorname{Tor}_{*}^{R}\left(M_{R}, S\right)$ and cannot therefore in general be taken as a lifting $\tilde{F}$ of $F$. However, only the initial part

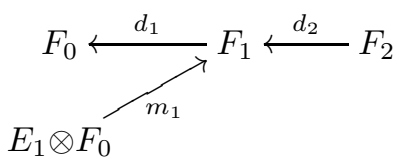


where $d_{1} m_{1}$ equals the map induced by the multiplication by (generators of) $J_{S}$ on $F$, is essential to the existence of liftings to $A_{R}$ and to $B_{R}$ (as will be exploited later on). The $\mathrm{o}_{A_{S}}$ is induced by $\tilde{d}_{1} \tilde{d}_{2}$ and if $\mathrm{o}_{A_{S}}=0$ modify $\tilde{d}_{1}$ and $\tilde{d}_{2}$ by $\tau_{1}$ and $\tau_{2}$ as before. $M_{R}=\mathrm{H}_{0}(\tilde{F})$ has $M_{R} \otimes_{R} S=M$. In a resolution $F_{R}$ for $M_{R}$ we can choose $\left(F_{R}\right)_{i}=\tilde{F}_{i}$ for $i=0,1$ and $\left(F_{R}\right)_{2}=\tilde{F}_{2} \oplus K_{2}$. Then the Tor-condition follows:

$$
\operatorname{Tor}_{1}^{R}\left(M_{R}, S\right)=\mathrm{H}_{1}\left(F_{R} \otimes_{R} S\right)=\frac{\operatorname{ker}\left(F_{1} \longrightarrow F_{0}\right)}{\operatorname{im}\left(F_{2} \oplus\left(K_{2} \otimes_{R} S\right) \longrightarrow F_{1}\right)}=0,
$$

so $M_{R}=\mathrm{H}_{0}(\tilde{F})$ is certainly a lifting. The $\mathrm{o}\left(J_{S}\right)$ is defined as induced by $\tilde{d}_{1} \tilde{m}_{1}-m_{E_{1}}$, where $m_{E_{1}}: E_{1} \otimes_{A} \tilde{F}_{0} \rightarrow \tilde{F}_{0}$ is induced by the multiplication of $J_{R}$ on $\tilde{F}_{0}$. The rest follows as above.

Remark 4 . We shall primarily be interested in the deformation situation, Definition 1 and in the case of a small lifting situation, Definition 2. If $M_{S}$ is a deformation of $M$ to $S$ in $\operatorname{Art}_{\mathcal{O}}$ one has natural isomorphisms like $\operatorname{Ext}_{A_{S}}^{i}\left(M_{S}, M_{S} \otimes_{S} I\right) \cong$ $\operatorname{Ext} \frac{i}{A}(M, M) \otimes_{k} I$ and $\operatorname{Hom}_{A_{S}}\left(J_{S} \otimes_{A_{S}} M_{S}, M_{S} \otimes_{S} I\right) \cong \operatorname{Hom}_{\bar{A}}\left(\bar{J} \otimes_{\bar{A}} M, M\right) \otimes_{k} I$ because $I \cdot \mathfrak{m}_{S}=0$ and therefore $M_{S} \otimes_{S} I \cong M_{S} \otimes_{S} k \otimes_{k} I \cong M \otimes_{k} I$, and because the spectral sequence $\mathrm{E}_{2}^{p q}=\operatorname{Ext}_{\bar{A}}\left(\operatorname{Tor}_{q}^{A_{S}}\left(M_{S}, \bar{A}\right), N\right) \Rightarrow \operatorname{Ext}_{A_{S}}^{p+q}\left(M_{S}, N\right)$ degenerates to $\operatorname{Ext} \frac{p}{A}(M, N) \cong \operatorname{Ext}_{A_{S}}^{p}\left(M_{S}, N\right)$ since $\operatorname{Tor}_{q}^{A_{S}}\left(M_{S}, \bar{A}\right) \cong \operatorname{Tor}_{q}^{S}\left(M_{S}, k\right)=0$ for $q>0$. The existence of such constant groups (like $\operatorname{Ext}_{\bar{A}}(M, M)$ and $\operatorname{Hom}_{\bar{A}}\left(\bar{J}_{\bar{A}_{A}} M, M\right)$ ) is essential for the existence of an obstruction algorithm. With a fixed $k$-basis the constant cohomology groups will keep track of the different obstruction "polynomials" in (the varying) $I$. To simplify the notation in the deformation situation, let $\mathrm{o}_{A_{S}}=\mathrm{o}_{A}, \mathrm{o}_{J_{S}}=\mathrm{o}_{J}$ and so on.

Example 3. A matrix factorisation (mf) of an element $f$ in a ring $A$ is a pair $(\varphi, \psi)$ of maps of free modules $\varphi: F \rightarrow G, \psi: G \rightarrow F$ with $\varphi \psi=f \cdot \operatorname{id}_{G}$ and $\psi \varphi=f \cdot \operatorname{id}_{F}$. Let $B=A /(f)$. Then $M=\operatorname{coker} \varphi$ is a $B$-module as $A$-module since $f$ annihilates $M$. If $f$ is $A$-regular, then the following 2-periodic complex of free $B$-modules (necessarily of equal $\operatorname{rank}$ if $A$ is Noetherian and $\operatorname{rk} G<\infty$ )

$$
\bar{G} \stackrel{\bar{\varphi}}{\longleftarrow} \stackrel{\bar{\psi}}{\longleftarrow} \bar{G} \stackrel{\bar{\varphi}}{\longleftarrow} \stackrel{\bar{\psi}}{\longleftarrow} \ldots
$$

is a free resolution of $M$, where $\bar{F}=F \otimes_{A} B$, etc. Maximal Cohen-Macaulay modules over a hypersurface singularity are given by $\mathrm{mfs}$ of the hypersurface. Mfs were introduced by D. Eisenbud in [4. A deformation of $M$ as a $B$-module will be given by a lifting of this resolution. One will therefore have conditions for lifting the equations $\bar{\varphi} \bar{\psi}=0$ which create the obstruction $\mathrm{o}_{B}$ in $\operatorname{Ext}_{B}^{2}(M, M)$. Instead Theorem 1 offers the possibility of lifting $\varphi$ corresponding to deformations of $M$ as an $A$-module for which the obstruction $\mathrm{o}_{A}=0$ since $\operatorname{Ext}_{A}^{i}(M, M)=0$ for $i>1$, such that there is a lifting of $\psi$ retaining the relation $\varphi \psi=f \cdot \mathrm{id}_{G}$. This gives the non-trivial obstruction $\mathrm{O}_{J}($ with $J=(f))$ in the cokernel of $\partial_{J}: \operatorname{Ext}_{A}^{1}(M, M) \rightarrow \operatorname{End}_{A}(M)$ where $\partial_{J}=\psi^{*}$; see Definition 3 Even in this most simple example the advantages are clear: The $A$-cohomology is easier than the $B$-cohomology and the relation $f=0$ is eliminated from the obstruction calculus. Further simplifications are possible in the case $\operatorname{rk}_{B}(M)=1$ as we show in $[9]$. 


\section{The obstruction maps}

We define obstruction maps $\mathrm{O}^{A}$ and $\mathrm{o}^{J}$ for the obstructions $\mathrm{o}_{A}$ and $\mathrm{o}_{J}$ in Definition 5 and give a structure theorem for the hull of $\operatorname{Def}_{M}^{B}$ in Theorem 2, A comparison of the $A$ - and $J$-obstruction maps with the $B$-obstruction map is given in Theorem 3

For greater flexibility we will define obstruction maps as continuous maps between local $\mathcal{O}$-algebras which have countably dimensional Zariski tangent spaces. Let $V$ be a countably-dimensional vector space over $k$ with a given basis $\left\{e_{i}\right\}_{i=1}^{\infty}$. The set of sub-vector spaces of $V$ which contain almost all the basis elements defines a topology on $V$ such that $V^{*}=\operatorname{Hom}_{k \text {-vec. }}^{\text {cont. }}(V, k)$ also is countably dimensional, and if we fix the dual basis $\left\{e_{i}^{*}\right\}_{i=1}^{\infty}$ for $V^{*}$, then $V^{* *} \cong V$ canonically. Let $T=\widehat{\text { Free }} \mathcal{O}\left(V^{*}\right)$ be the free $\mathcal{O}$-algebra in variables $\left\{x_{i}\right\}_{i=1}^{\infty}$ completed in the topology given by the basis $\mathcal{I}_{i j l}$ of open ideals around 0 , where $\mathcal{I}_{i j l}=$ $\mathfrak{m}_{\mathcal{O}}^{i} T+\left(x_{1}, x_{2}, \ldots\right)^{j}+\left(x_{l}, x_{l+1}, \ldots\right)$. We insist on the continuous identification of the relative cotangent vector space $\mathfrak{m} /\left(\mathfrak{m}^{2}+\mathfrak{m}_{\mathcal{O}} T\right)$ of $T$ with $V^{*}$ where $\bar{x}_{i}=e_{i}^{*}$, hence also a canonical continuous identification of the relative Zariski tangent space of $T$ with $V$. Suppose $\left\{H_{n}\right\}_{n=1}^{\infty}$ is a projective system of surjections in Art $_{\mathcal{O}}$. Then $H:=\lim _{n} H_{n}$ with the induced topology is a continuous quotient of a $T$ for some $V$. Conversely every continuous quotient of $T$ can be given as such a projective limit. Define $\widehat{D}_{M}^{B}(H)=\lim _{\leftarrow} \operatorname{Def}_{M}^{B}\left(H_{n}\right)$.

Recall the map $\overleftarrow{\partial_{J}}$ with $N=M$; see Definition [3. Assume for the rest of this section that the $k$-vector spaces $\mathrm{H}_{A}^{2}=\operatorname{im}\left(\operatorname{Ext}_{\bar{B}}(M, M) \rightarrow \operatorname{Ext}_{\frac{2}{A}}(M, M)\right)$, $\mathrm{H}_{J}^{2}=\operatorname{coker} \partial_{\bar{J}}$ and $\mathrm{H}^{1}=\operatorname{ker} \partial_{\bar{J}}$ all are of countable $k$-dimension and for any choice of $k$-bases let $T_{A}^{2}, T_{J}^{2}$ and $T^{1}$ be the corresponding complete $\mathcal{O}$-algebras with these vector spaces as relative Zariski tangent spaces.

Definition 5. In the situation described before Lemma 1 two obstruction maps for the obstructions $\mathrm{O}_{A}$ and $\mathrm{O}_{J}$ in Theorem 1 (see Remark 4 ) are continuous $\mathcal{O}$ algebra homomorphisms $\mathrm{O}^{A}: T_{A}^{2} \rightarrow T^{1}$ and $\mathrm{O}^{J}: T_{J}^{2} \rightarrow T^{1}$ satisfying the following conditions. If $H:=\left(T^{1} \hat{\otimes}_{T_{A}^{2}} \mathcal{O}\right) \hat{\otimes}_{T_{J}^{2}} \mathcal{O}$ there is a formal deformation $\widehat{M}$ in $\widehat{D}_{M}^{B}(H)$ such that for any small lifting situation, Definition 2 there is a continuous $\sigma$ : $H \rightarrow S$ with $\sigma_{*} \widehat{M}=M_{S}$ and for any such $\sigma$ we have that the adjoint $\mathrm{o}_{A}^{\text {adj }}$ of $\mathrm{o}_{A}\left(\pi, M_{S}\right) \in \mathrm{H}_{A}^{2} \otimes_{k} I$ makes the following diagram commutative:

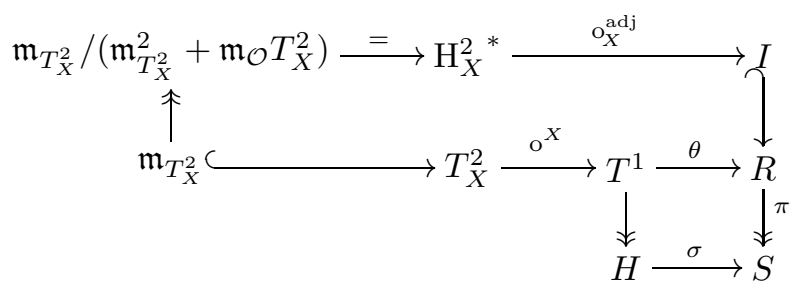

where $\theta$ is continuous and lifts $\sigma$ and $X=A$. If $\mathrm{o}_{A}\left(\pi, M_{S}\right)=0$, then the adjoint $\mathrm{o}_{J}^{\text {adj }}$ of $\mathrm{o}_{J}\left(\pi, M_{S}\right) \in \mathrm{H}_{J}^{2} \otimes_{k} I$ makes the diagram commutative with $X=J$.

Remark 5. The existence of two obstruction maps as in Definition 5 implies that $(H, \widehat{M})$ is a hull of $\operatorname{Def}_{M}^{B}$; see the last part of the proof of Theorem 2 .

The following theorem connects the cohomologically defined and thus linear obstruction classes in Theorem 1 with the highly non-linear obstruction ideal. The proof shows how the former are used to construct the latter. 
Theorem 2. Let $A$ and $B$ be flat $\mathcal{O}$-algebras with $B=A / J$ for an ideal $J \subset A$. Let $M$ be a $\bar{B}=\bar{A} / \bar{J}$-module, where $\bar{X}=X \otimes_{\mathcal{O}} k$ for $X=A, B$ and $J$. Then $\operatorname{Def}_{M}^{B}$ is a functor with two obstructions in $\mathrm{H}_{A}^{2}$ and $\mathrm{H}_{J}^{2}$ such that if $\mathrm{H}^{1}, \mathrm{H}_{A}^{2}$ and $\mathrm{H}_{J}^{2}$ have countable $k$-dimension, there are obstruction maps

$$
\mathrm{o}^{A}: T_{A}^{2} \longrightarrow T^{1} \quad \text { and } \quad \mathrm{o}^{J}: T_{J}^{2} \longrightarrow T^{1}
$$

for the obstructions $\mathrm{O}_{A}$ and $\mathrm{O}_{J}$. In particular the hull of $\operatorname{Def}_{M}^{(A, J)} \cong \operatorname{Def}_{M}^{B}$ is given as

$$
H \cong\left(T^{1} \hat{\otimes}_{T_{A}^{2}} \mathcal{O}\right) \hat{\otimes}_{T_{J}^{2}} \mathcal{O} .
$$

Remark 6 . The statement implicitly claims the existence of $k$-vector bases and hence topologies as described before Definition 5 and maps continuous with respect to these topologies.

Remark 7. If $\bar{A}$ is a finitely generated $k$-algebra and $M$ is a finitely generated $\bar{A}$ module, then the $\operatorname{Ext} \frac{i}{A}(M, M)$ have countable $k$-dimensions since they are finitely generated as $\bar{A}$-modules and $\bar{A}$ has countable $k$-dimension. It follows that $\mathrm{H}^{1}, \mathrm{H}_{A}^{2}$ and $\mathrm{H}_{J}^{2}$ have countable $k$-dimensions.

Proof. Assume first that $\operatorname{dim}_{k} \mathrm{H}^{1}<\infty$. Let $T_{n}^{1}=T^{1} / \mathfrak{m}^{n-1} \mathfrak{n}$, where $\mathfrak{n}=\mathfrak{m}^{2}+$ $\mathfrak{m}_{\mathcal{O}} T^{1}$. The universal extension (11) gives a canonical deformation $\pi_{1}: M_{1} \rightarrow M$ of $M$ to $T_{1}^{1}$ as a $B$-module. There is no obstruction for doing this, thus $\left(\mathrm{o}_{1}^{A}, \mathrm{o}_{1}^{J}\right)$ are the trivial maps. We construct approximations $\left(\mathrm{o}_{n}^{A}, \mathrm{O}_{n}^{J}\right)$ to $\left(\mathrm{o}^{A}, \mathrm{o}^{J}\right)$ by induction on $n$. Suppose $\mathrm{o}_{n-1}^{A}\left(y_{i}\right)=f_{i}^{n-1}, \mathrm{o}_{n-1}^{J}\left(z_{j}\right)=g_{j}^{n-1}$ in $T^{1}$ for all $i, j$, where $T_{A}^{2}=$ $\mathcal{O}\left[y_{1}, y_{2}, \ldots\right]^{\wedge}, T_{J}^{2}=\mathcal{O}\left[z_{1}, z_{2}, \ldots\right]^{\wedge}$ such that there is a deformation $M_{n-1}$ of $M$ to $H_{n-1}=T_{n-1}^{1} /\left(\bar{f}_{i}^{n-1}\right)+\left(\bar{g}_{j}^{n-1}\right)$ as $B$-module lifting $M_{1}$. Define the A-obstruction test algebra $G_{n}^{A}=T_{n}^{1} /\left(\bar{f}_{i}^{n-1}\right) \mathfrak{m}+\left(\bar{g}_{j}^{n-1}\right) \mathfrak{m}$ and let $\pi_{n}^{A}: G_{n}^{A} \rightarrow H_{n-1}$ be the natural map. If $\pi: R \rightarrow S$ and $M_{S}$ is a small lifting situation, Definition 2] with $\mathfrak{m}_{S}^{n-2} \mathfrak{n}_{S}=$ 0 and $\theta: T^{1} \rightarrow R$ lifts a map $\sigma: H_{n-1} \rightarrow S$ as in (6), then $\theta$ factorises through $G_{n}^{A}$. Let $I_{n}^{A}=\operatorname{ker} \pi_{n}^{A}$ which is a $k$-vector space as a $G_{n}^{A}$-module. By induction it will follow that $I_{n}^{A}=\left(\bar{f}_{i}^{n-1}\right) \oplus\left(\bar{g}_{j}^{n-1}\right) \oplus \mathfrak{m}^{n-2} \mathfrak{n} \subseteq G_{n}^{A}$, and the elements $\left\{\bar{f}_{i}^{n-1}, \bar{g}_{j}^{n-1}\right\}_{i, j}$ can be assumed to be linearly independent. There is a natural map $G_{n}^{A} \rightarrow G_{n-1}^{A}$ lifting $H_{n-1} \rightarrow H_{n-2}$, by the naturality of the obstruction class $\mathrm{o}_{A}$, Theorem 1 $\mathrm{o}_{A}\left(\pi_{n}^{A}, M_{n-1}\right)=\sum y_{i}^{*} \otimes \bar{f}_{i}^{n} \in \mathrm{H}_{A}^{2} \otimes_{k} I_{n}^{A}$ maps to

$$
\mathrm{o}_{A}\left(\pi_{n-1}^{A}, M_{n-2}\right)=\sum y_{i}^{*} \otimes\left(\bar{f}_{i}^{n-2}+\bar{f}_{i}^{(n-1)}\right) \in \mathrm{H}_{A}^{2} \otimes_{k} I_{n-1}^{A},
$$

where $\bar{f}_{i}^{n-1} \mapsto \bar{f}_{i}^{n-2}+\bar{f}_{i}^{(n-1)}$ with $\bar{f}_{i}^{(n-1)} \in \mathfrak{m}^{n-3} \mathfrak{n}$ under the (assumed) injective $\operatorname{map} I_{n}^{A} \supseteq\left(\bar{f}_{i}^{n-1}\right) \oplus\left(\bar{g}_{j}^{n-1}\right) \hookrightarrow I_{n-1}^{A}$. Hence $\bar{f}_{i}^{n}=\bar{f}_{i}^{n-1}+\bar{f}_{i}^{(n)}$ for some $\bar{f}_{i}^{(n)} \in \mathfrak{m}^{n-2} \mathfrak{n}$. Choose a lifting $f_{i}^{(n)} \in \mathfrak{m}^{n-2} \mathfrak{n} \subseteq T^{1}$ of $\bar{f}_{i}^{(n)}$ and set $\mathrm{o}_{n}^{A}\left(y_{i}\right)=f_{i}^{n}=f_{i}^{n-1}+f_{i}^{(n)}$ for all $i$.

Define the $J$-obstruction test algebra $G_{n}^{J}=T_{n}^{1} /\left(\bar{f}_{i}^{n}\right)+\left(\bar{g}_{j}^{n-1}\right) \mathfrak{m}$ and let $\pi_{n}^{J}$ : $G_{n}^{J} \rightarrow H_{n-1}$ be the natural map. Suppose $\mathrm{o}_{A}\left(\pi, M_{S}\right)=0$. Since $\mathrm{o}_{A}$ is natural and $\mathrm{o}_{A}\left(\pi_{n}^{A}, M_{n-1}\right) \in \mathrm{H}_{A}^{2} \otimes_{k} I_{n}^{A}$ maps to 0 in $\mathrm{H}_{A}^{2} \otimes_{k} \operatorname{ker} \pi, \bar{f}_{i}^{n} \mapsto 0$ and the induced map $G_{n}^{A} \rightarrow R$ factorises through $G_{n}^{J}$. Let $I_{n}^{J}=\operatorname{ker} \pi_{n}^{J}$. By induction it will follow that $I_{n}^{J}=\left(\bar{g}_{j}^{n-1}\right) \oplus \mathfrak{m}^{n-2} \mathfrak{n} \subseteq G_{n}^{J}$. There is a natural map $G_{n}^{J} \rightarrow G_{n-1}^{J}$ lifting $H_{n-1} \rightarrow$ $H_{n-2}$, by the naturality of the obstruction class $\mathrm{o}_{J}, \mathrm{o}_{J}\left(\pi_{n}^{J}, M_{n-1}\right)=\sum z_{j}^{*} \otimes \bar{g}_{j}^{n} \in$ $\mathrm{H}_{J}^{2} \otimes_{k} I_{n}^{J}$ maps to $\mathrm{o}_{J}\left(\pi_{n-1}^{J}, M_{n-2}\right)=\sum z_{j}^{*} \otimes\left(\bar{g}_{j}^{n-2}+\bar{g}_{j}^{(n-1)}\right) \in \mathrm{H}_{J}^{2} \otimes_{k} I_{n-1}^{J}$, and as 
above it follows that $\bar{g}_{j}^{n}=\bar{g}_{j}^{n-1}+\bar{g}_{j}^{(n)}$ for some $\bar{g}_{j}^{(n)} \in \mathfrak{m}^{n-2} \mathfrak{n} \subseteq G_{n}^{J}$, lifting to $T^{1}$ gives $\mathrm{o}_{n}^{J}\left(z_{j}\right)=g_{j}^{n}=g_{j}^{n-1}+g_{j}^{(n)}$ with $g_{j}^{(n)} \in \mathfrak{m}^{n-2} \mathfrak{n} \subseteq T^{1}$. Let $H_{n}=T_{n}^{1} /\left(\bar{f}_{i}^{n}\right)+\left(\bar{g}_{j}^{n}\right)$, if $\pi_{n}: H_{n} \rightarrow H_{n-1}$ is the natural map, $\mathrm{o}_{A}\left(\pi_{n}, M_{n-1}\right)=0=\mathrm{o}_{J}\left(\pi_{n}, M_{n-1}\right)$, hence by Theorem 1 there is a lifting $M_{n}$ to $H_{n}$ of $M_{n-1}$.

Remark that $M_{n}$ is $H_{n}$-flat and thus gives a deformation of $M$ to $H_{n}$ as $B$-module lifting $M_{n-1}$ : There is a change of rings spectral sequence

$$
\mathrm{E}_{p q}^{2}=\operatorname{Tor}_{p}^{H_{n-1}}\left(\operatorname{Tor}_{q}^{H_{n}}\left(M_{n}, H_{n-1}\right), N\right) \Rightarrow \operatorname{Tor}_{p+q}^{H_{n}}\left(M_{n}, N\right),
$$

and the 5 -term exact sequence

$$
\ldots \rightarrow \operatorname{Tor}_{1}^{H_{n}}\left(M_{n}, H_{n-1}\right) \otimes_{H_{n-1}} N \longrightarrow \operatorname{Tor}_{1}^{H_{n}}\left(M_{n}, N\right) \longrightarrow \operatorname{Tor}_{1}^{H_{n-1}}\left(M_{n-1}, N\right) \rightarrow 0
$$

implies that $\operatorname{Tor}_{1}^{H_{n}}\left(M_{n}, N\right)=0$ for any $H_{n-1}$-module $N$ since $\operatorname{Tor}_{1}^{H_{n}}\left(M_{n}, H_{n-1}\right)=$ 0 by assumption and $M_{n-1}$ is $H_{n-1}$-flat by induction. If $N$ is any $H_{n}$-module, there is a short exact sequence of $H_{n}$-modules $0 \rightarrow N I \rightarrow N \rightarrow N / N I \rightarrow 0$ with $I=\operatorname{ker}\left(H_{n} \rightarrow H_{n-1}\right)$, where both $N I$ and $N / N I$ are $H_{n-1}$-modules as $H_{n^{-}}$ modules, hence we have $\operatorname{Tor}_{1}^{H_{n}}\left(M_{n}, N\right)=0=\operatorname{Tor}_{1}^{H_{n}}\left(M_{n}, N / N I\right)$ which implies $\operatorname{Tor}_{1}^{H_{n}}\left(M_{n}, N\right)=0$, so $M_{n}$ is $H_{n}$-flat.

To show that we may assume $I_{n+1}^{A}=\left(\bar{f}_{i}^{n}\right) \oplus\left(\bar{g}_{j}^{n}\right) \oplus \mathfrak{m}^{n-1} \mathfrak{n}$ with $\left\{\bar{f}_{i}^{n}, \bar{g}_{j}^{n}\right\}_{i, j}$ a $k$ linearly independent set, let $\varphi_{n}^{A}:\left(I_{n}^{A}\right)^{*} \rightarrow \mathrm{H}_{A}^{2}$ correspond to $\mathrm{o}_{A}\left(\pi_{n}^{A}, M_{n-1}\right)$ under the natural $k$-isomorphism $\operatorname{Hom}_{k}\left(\left(I_{n}^{A}\right)^{*}, \mathrm{H}_{A}^{2}\right) \cong \mathrm{H}_{A}^{2} \otimes_{k} I_{n}^{A}$. Supplement, if necessary, the by induction chosen $k$-linearly independent set $\left\{y_{1}^{*}, \ldots, y_{r_{n-1}}^{*}\right\}$ so that $\left\{y_{1}^{*}, \ldots, y_{r_{n}}^{*}\right\}$ gives a $k$-basis for $\operatorname{im} \varphi_{n}^{A} \subseteq \mathrm{H}_{A}^{2}$. Then there can be no $k$-relations among the $\bar{f}_{i}^{n}$, where $\mathrm{o}_{A}\left(\pi_{n}^{A}, M_{n-1}\right)=\sum_{i=1}^{r_{n}} y_{i}^{*} \otimes \bar{f}_{i}^{n}$. The $z_{j}^{*}$ are chosen similarly to give a $k$-basis for $\operatorname{im} \varphi_{n}^{J} \subseteq \mathrm{H}_{J}^{2}$, and $\left\{\bar{f}_{i}^{n}, \bar{g}_{j}^{n}\right\}$ is also a $k$-linearly independent set as the $\bar{g}_{j}^{n}$ are contained in an orthogonal complement of $\left(\bar{f}_{i}^{n}\right)$ by construction. Finally, if $\sum \alpha_{i} \bar{f}_{i}^{n}+\sum \beta_{j} \bar{g}_{j}^{n} \in \mathfrak{m}^{n-1} \mathfrak{n}$, then the image in $G_{n}^{A}$ gives $\sum \alpha_{i} \bar{f}_{i}^{n}+\sum \beta_{j} \bar{g}_{j}^{n}=0$ and $\alpha_{i}=0=\beta_{j}$. This also implies that the restriction of $I_{n+1}^{A} \rightarrow I_{n}^{A}$ to $\left(\bar{f}_{i}^{n}\right) \oplus\left(\bar{g}_{j}^{n}\right) \subseteq I_{n+1}^{A}$ is an inclusion, and analogously $\left(\bar{g}_{j}^{n}\right) \hookrightarrow I_{n}^{J}$.

By induction we have constructed $f_{i}^{(n)}, g_{j}^{(n)} \in \mathfrak{m}^{n-2} \mathfrak{n} \subseteq T^{1}$ for all $n \geqslant 2$. Let $\mathrm{o}^{A}\left(y_{i}\right)=f_{i}=\sum_{n \geqslant 2} f_{i}^{(n)}$ and $\mathrm{o}^{J}\left(z_{j}\right)=g_{j}=\sum_{n \geqslant 2} g_{j}^{(n)}$; they give well defined elements in $T^{1}$, put $H=T^{1} /\left(f_{i}\right)+\left(g_{j}\right)$ and $\widehat{M}=\left\{M_{n}\right\}$, then $H \cong \lim H_{n}$.

For the general case, choose a sequence of finite-dimensional $k$-vector spaces $V_{1} \subset V_{2} \subset \ldots \subset \mathrm{H}^{1}$ with $\bigcup V_{s}=\mathrm{H}^{1}$. This gives a topology on $\mathrm{H}^{1}$ as described before Definition 5 and hence on $\mathrm{H}^{1^{*}}=\lim _{\longleftarrow} V_{s}^{*}$ and on $T^{1}=\lim _{s} T^{1}$, where ${ }_{s} T^{1}$ has $V_{s}$ as relative Zariski tangent space. For each $s$ the construction above beginning with the extension

$$
{ }_{s} M_{1}: \quad 0 \rightarrow M \otimes_{k} V_{s}^{*} \longrightarrow{ }_{s} M_{1} \stackrel{{ }^{s} \pi_{1}}{\longrightarrow} M \rightarrow 0
$$

is applied to produce a pair $\left({ }_{s} \mathrm{O}^{A},{ }_{s} \mathrm{O}^{J}\right),{ }_{s} \mathrm{O}^{A}: T_{A}^{2} \rightarrow{ }_{s} T^{1},{ }_{s} \mathrm{O}^{J}: T_{J}^{2} \rightarrow{ }_{s} T^{1}$ and $\left\{{ }_{s} M_{n}\right\}$. By induction on $s$ and $n$ we will show that there is a choice of $\left(s_{s+1} \mathrm{O}^{A},{ }_{s+1} \mathrm{O}^{J}\right)$ which lifts $\left({ }_{s} \mathrm{O}^{A},{ }_{s} \mathrm{O}^{J}\right)$ over ${ }_{s+1} T^{1} \rightarrow{ }_{s} T^{1}$ : By naturality of the obstruction class, $\mathrm{o}_{A}\left(s_{s+1} \pi_{n}^{A},{ }_{s+1} M_{n-1}\right)=\sum y_{i}^{*} \otimes_{s+1} \bar{f}_{i}^{n} \in \mathrm{H}_{J}^{2} \otimes_{k}\left({ }_{s+1} I_{n}^{A}\right)$ maps to $\mathrm{o}_{A}\left({ }_{s} \pi_{n}^{A},{ }_{s} M_{n-1}\right)=$ $\sum y_{i}^{*} \otimes_{s} \bar{f}_{i}^{n} \in \mathrm{H}_{J}^{2} \otimes_{k}\left({ }_{s} I_{n}^{A}\right)$ under the map ${ }_{s+1} G_{n}^{A} \rightarrow{ }_{s} G_{n}^{A}$. A $k$-linear section $\sigma$, $V_{s+1}^{*} \longleftarrow V_{s}^{*}$, induces a filtration preserving section ${ }_{s+1} T^{1} \coprod_{s} T^{1}$. A set of elements in $\mathfrak{m}^{n-2} \mathfrak{n} \subseteq{ }_{s} T^{1}$ inducing a $k$-basis for $\mathfrak{m}^{n-2} \mathfrak{n} \subseteq{ }_{s} G_{n}^{A}$ can be viewed as 
elements in $\mathfrak{m}^{n-2} \mathfrak{n} \subseteq{ }_{s+1} T^{1}$ via the section and they can be supplemented to a set which induces a $k$-basis for $\mathfrak{m}^{n-2} \mathfrak{n} \subseteq{ }_{s+1} G_{n}^{A}$. This set enables us to choose liftings ${ }_{s+1} f_{i}^{(n)}$ of ${ }_{s+1} \bar{f}_{i}^{(n)}$ mapping to ${ }_{s} f_{i}^{(n)}$ under ${ }_{s+1} T^{1} \rightarrow{ }_{s} T^{1}$. Similar arguing ensures the existence of ${ }_{s+1} g_{j}^{(n)} \in{ }_{s+1} T^{1}$ mapping to both ${ }_{s+1} \bar{g}_{j}^{(n)}$ and to ${ }_{s} g_{j}^{(n)}$. If new obstruction elements $y_{i}^{*}$ and $z_{j}^{*}$ are necessary, they should be chosen to give $k$-bases for $\operatorname{im}\left(s+1 \varphi_{n}^{A}\right) \subseteq \mathrm{H}_{A}^{2}$ and for $\operatorname{im}\left(s+1 \varphi_{n}^{J}\right) \subseteq \mathrm{H}_{J}^{2}$ as above.

We want to find a deformation ${ }_{s+1} M_{n}$ to ${ }_{s+1} H_{n}$ lifting both ${ }_{s+1} M_{n-1}$ and ${ }_{s} M_{n}$. Choose any lifting ${ }_{s+1} M_{n}^{\prime}$ of ${ }_{s+1} M_{n-1}$. Let $\psi:{ }_{s+1} H_{n} \rightarrow{ }_{s} H_{n}$ be the natural map, then, essentially by Theorem 1 1iv) and induction, there is a $\xi \in V_{s} \otimes_{k}\left({ }_{s} I_{n}\right)$, where ${ }_{s} I_{n}=\operatorname{ker}\left({ }_{s} H_{n} \rightarrow{ }_{s} H_{n-1}\right)$, which gives the difference of $\psi_{*}\left({ }_{s+1} M_{n}^{\prime}\right)$ and ${ }_{s} M_{n}$. Perturbing ${ }_{s+1} M_{n}^{\prime}$ by a lifting $\tilde{\xi} \in V_{s} \otimes_{k}\left({ }_{s+1} I_{n}\right) \rightarrow V_{s} \otimes_{k}\left({ }_{s} I_{n}\right)$ of $\xi$, gives our desired ${ }_{s+1} M_{n}$.

Let $H_{n}={ }_{n} H_{n}$ and $M_{n}={ }_{n} M_{n}$. Then $H \cong \lim _{\longleftarrow} H_{n}$ (by a continuous isomorphism of $\mathcal{O}$-algebras), where $H$ is defined by $\mathrm{O}^{A}=\lim ^{\longleftarrow}\left\{{ }_{n} \mathrm{O}_{n}^{A}\right\}$ and $\mathrm{O}^{J}=\lim _{n}\left\{{ }_{n} \mathrm{O}_{n}^{J}\right\}$.

To prove smoothness [19, 2.2] of $\operatorname{Hom}_{\mathcal{O}-\text { alg. } / k}^{\text {cont. }}(H,-) \rightarrow \operatorname{Def}_{M}^{B}$, suppose $\pi: R \rightarrow$ $S, M_{S}$ is a small lifting situation and assume there is a deformation $M_{R}$ of $M$ to $R$ lifting $M_{S}$. Moreover assume there is an $n$ and a continuous map $\sigma: H_{n} \rightarrow S$ with $\sigma_{*} M_{n} \cong M_{S}$. Choose any $\theta: T^{1} \rightarrow R$ lifting $\sigma$. Then by Theorem 1 and the construction of $H_{n+1}, \theta$ factorises through $\bar{\theta}: H_{n+1} \rightarrow R$. By Theorem 1iv) there exists an $m \geqslant n+1$ and a $\xi \in V_{m} \otimes_{k} \operatorname{ker} \pi$ which gives the difference of $\bar{\theta}_{*} M_{n+1}$ and $M_{R}$. If we "add" the adjoint $\xi^{a}: V_{m}^{*} \rightarrow \operatorname{ker} \pi$ to the pullback $\theta_{m}: H_{m} \rightarrow R$ of $\bar{\theta}$, we obtain a map $\theta_{m}^{\prime}: H_{m} \rightarrow R$ which lifts $\sigma$ and such that $\left(\theta_{m}^{\prime}\right)_{*} M_{m} \cong M_{R}$. Finally $\operatorname{Hom}_{\mathcal{O}-\text { alg. } / k}^{\text {cont. }}(H, k[\varepsilon]) \cong \mathrm{H}^{1} \cong \operatorname{Def}_{M}^{B}(k[\varepsilon])$ by Theorem 11 and $\left(H,\left\{M_{n}\right\}\right)$ gives a hull for $\operatorname{Def}_{M}^{B}$. In particular this implies that $\operatorname{Hom}_{\mathcal{O}-\text { alg. } / k}^{\text {cont. }}(H, S) \rightarrow \operatorname{Def}_{M}^{B}(S)$ is surjective for all $S$ in $\operatorname{Art}_{\mathcal{O}}$ and all the conditions in Definition 5 are fulfilled.

Example 4. Remark that every $\mathcal{O}$-algebra in the pro-category of $A t_{\mathcal{O}}$ is obtained as the hull of the deformation functor of a module. In fact the following argument is valid for the non-commutative deformation functor of modules as well as for the commutative one. In the non-commutative case $\operatorname{Art}_{\mathcal{O}}$ is the category of local not necessarily commutative Artinian $\mathcal{O}$-algebras $(\mathcal{O}$ as in Definition 11) $R$ with $k$ as residue field (i.e. $k$ is the unique simple $R$-module). $A$ and $B$ may also be noncommutative $\mathcal{O}$-algebras. A deformation of a left $\bar{A}$-module $M$ is defined as in Definition 1 except that $M_{R}$ is an $A-R$-bimodule which is a left $A$-module and a right $R$-module, or equivalent, a left $A \otimes_{\mathcal{O}} R^{\circ}$-module. Furthermore, $\widehat{\operatorname{Fr}} \operatorname{ce}_{\mathcal{O}}\left(V^{*}\right)$ is the free non-commutative $\mathcal{O}$-algebra, completed in the topology defined by ideals $\mathcal{I}_{i j l}$ analogous to the ones in the beginning of this section, e.g. where the "power ideal" $\left(x_{1}, x_{2}, \ldots\right)^{j}$ is replaced by the (two-sided) ideal generated by $j$-tensors, and so on.

Fix a maximal ideal $\mathfrak{m}$ in an $\mathcal{O}$-algebra $B$ such that $B / \mathfrak{m} \cong k$ and assume $B /\left(\mathfrak{m}^{2}+\mathfrak{m}_{\mathcal{O}} B\right)$ is countably dimensional. Let $\hat{B}$ be the completion of $B$ in any topology as given in the beginning of this section (or analogous in the non-commutative case). Then

$$
\operatorname{Hom}_{\mathcal{O}-\text { alg. } / k}^{\text {cont. }}(\hat{B},-) \stackrel{\simeq}{\longrightarrow} \operatorname{Def}_{k}^{B},
$$

where $\varphi \in \operatorname{Hom}_{\mathcal{O}-\text { alg. } / k}^{\text {cont. }}(\hat{B}, R)$ is mapped to the $B \otimes_{\mathcal{O}} R^{\mathrm{o}}$-module $R$ with module structure given by (left) multiplication of $B \otimes_{\mathcal{O}} R^{\mathrm{O}}$ through the composition 
$\hat{B} \otimes_{\mathcal{O}} R^{\mathrm{o}} \stackrel{\varphi \otimes \mathrm{id}}{\longrightarrow} R \otimes_{\mathcal{O}} R^{\mathrm{o}} \stackrel{\text { mult. }}{\longrightarrow} R^{\mathrm{o}}$. It gives a deformation of $k$ to $R$. For the inverse, any deformation $M_{R}$ of $k$ to $R$ has $M_{R} \cong R$ as $R$-modules since $M_{R}$ is $R$-flat, i.e. $R$-free of rank 1 . Hence $R$ has a (left) $B \otimes_{\mathcal{O}} R^{\circ}$-module structure. Define $\varphi: \hat{B} \rightarrow R$ by $\varphi(b):=(b \otimes 1) \bullet 1_{R}=r \in R$ for $b \in B$. Then $\varphi\left(b^{\prime} b\right)=b^{\prime} b \otimes 1 \bullet 1_{R}=$ $\left(b^{\prime} \otimes 1\right)(b \otimes 1) \bullet 1_{R}=\left(b^{\prime} \otimes 1\right) \bullet r=\left(b^{\prime} \otimes 1\right)(1 \otimes r) \bullet 1_{R}=(1 \otimes r)\left(b^{\prime} \otimes 1\right) \bullet 1_{R}=1 \otimes r \bullet r^{\prime}=$ $r^{\prime} r=\left(b^{\prime} \otimes 1 \bullet 1_{R}\right)\left(b \otimes 1 \bullet 1_{R}\right)=\varphi\left(b^{\prime}\right) \varphi(b), \varphi(1)=1_{R}$ and $\varphi$ is additive. If $f: \mathcal{O} \rightarrow B$ and $g: \mathcal{O} \rightarrow R$ define the $\mathcal{O}$-algebra structures, then $\varphi(f(\lambda) a)=f(\lambda) a \otimes 1 \bullet 1_{R}=$ $a \otimes g(\lambda) \bullet 1_{R}=(1 \otimes g(\lambda)) \bullet\left(a \otimes 1 \bullet 1_{R}\right)=g(\lambda)\left(a \otimes 1 \bullet 1_{R}\right)=g(\lambda) \varphi(a)$, hence $\varphi$ gives a well-defined $\mathcal{O}$-algebra homomorphism $\hat{B} \rightarrow R$ above $k$ and $\hat{B}$ pro-represents $\operatorname{Def}_{k}^{B}$. In particular

$$
\hat{B} \cong\left(T^{1} \hat{\otimes}_{T_{A}^{2}} \mathcal{O}\right) \hat{\otimes}_{T_{J}^{2}} \mathcal{O}
$$

for obstruction maps $o^{A}$ and $o^{J}$. For instance, if $B=\mathcal{O}$, then $\bar{B}=k$ and $\operatorname{Def}_{k}^{B}(R)$ is a one-element set for all $R$ and $\operatorname{Def}_{k}^{B}$ is pro-represented by $\mathcal{O}$.

Remark that if $B$ is non-commutative, we can still deform $k$ over commutative Artinian $\mathcal{O}$-algebras, but then the completion $\hat{B}$ will be in the ideals $\mathcal{I}_{i j l}+[B, B]$ and hence in that case be a commutative $\mathcal{O}$-algebra, indeed $\hat{B}=(B /[B, B])$.

In [8] we axiomatically define a functor with $n$ obstructions and corresponding obstruction maps and prove the existence of such maps in the countably-dimensional case. Theorem 2 is an instance of this. A single obstruction map defined for the obstruction $\mathrm{O}_{A}$ has been constructed by $\mathrm{O}$. A. Laudal in [15].

We next state a theorem describing how closely related the obstruction map o ${ }^{B}$, defined by $\bar{B}$-cohomology, is to $\mathrm{o}^{A}$ and $\mathrm{o}^{J}$, defined by $\bar{A}$-cohomology. Let $T_{B}^{2}$ be a local complete $\mathcal{O}$-algebra with relative Zariski tangent space $\operatorname{Ext} \frac{2}{B}(M, M)$ for any topology as in Theorem 2. In the next sections (Proposition 2 and Lemma 4) we show that there is a canonical isomorphism $\operatorname{ker} \partial_{\bar{J}} \cong \operatorname{Ext} \frac{1}{B}(M, M)$ and a natural exact sequence of $\bar{A}$-modules $0 \rightarrow \operatorname{coker} \partial_{\bar{J}} \rightarrow \operatorname{Ext} \frac{2}{B}(M, M) \rightarrow \operatorname{Ext} \frac{2}{A}(M, M)$. Hence there is a "short exact sequence" of continuous maps $T_{A}^{2} \hookrightarrow T_{B}^{2} \rightarrow T_{J}^{2}$. Our main comparison result reads:

Theorem 3. With assumptions as in Theorem Q given a pair of obstruction maps $\mathrm{o}^{A}: T_{A}^{2} \rightarrow T^{1}$ and $\mathrm{o}^{J}: T_{J}^{2} \rightarrow T^{1}$ for the obstructions $\mathrm{o}_{A}$ and $\mathrm{o}_{J}$, defining the hull of $\operatorname{Def}_{M}^{(A, J)}$, there exists an obstruction map $\mathrm{o}^{B}: T_{B}^{2} \longrightarrow T^{1}$ for the obstruction $\mathrm{O}_{B}$, defining the hull of $\operatorname{Def}_{M}^{B}$, such that

$$
\mathrm{o}^{B} \mid T_{A}^{2}=\mathrm{o}^{A}, \quad \text { and } \quad \mathrm{o}^{B} \hat{\otimes} \mathcal{O}=\mathrm{o}_{T_{A}^{J} \hat{\otimes} \mathcal{O}}^{2} \text { as maps } T_{J}^{2} \longrightarrow \underset{T_{A}^{2}}{\longrightarrow} T^{1} \hat{\otimes} \mathcal{O} .
$$

Conversely, given an obstruction map $\mathrm{O}^{B}$, there exists a pair of obstruction maps $\mathrm{O}^{A}$ and $\mathrm{O}^{J}$ such that the following diagram of continuous maps is commutative:

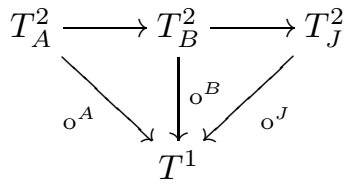

Remark 8. The $\mathrm{o}^{B}$-map is in general not the "union" of $\mathrm{o}^{A}$ and $\mathrm{o}^{J}$, but there is always a pair of obstruction maps $\left(\mathrm{o}^{A}, \mathrm{o}^{J}\right)$ such that $\mathrm{o}^{B}$ can be taken as the "union" of $\mathrm{O}^{A}$ and $\mathrm{O}^{J}$. 
We do not necessarily get a trivial $\mathrm{o}^{J_{1}+J_{2}}$ even if $\mathrm{o}^{J_{i}}$ is trivial for $i=1,2$. The reason for this is simply that the natural map coker $\partial_{J_{1}+J_{2}} \rightarrow \oplus$ coker $\partial_{J_{i}}$ does not have to be injective. An explicit example is given in [8] Ex. 4.1.4].

Remark 9. To distinguish it from $\mathrm{o}^{A}$ in the pair of obstruction maps $\left(\mathrm{o}^{A}, \mathrm{o}^{J}\right)$ for $\operatorname{Def}_{M}^{(A, J)}$, let $\mathrm{o}_{A}^{A}$ (in this remark) be an obstruction map

$$
\mathrm{o}_{A}^{A}: \widehat{\operatorname{Free}}_{\mathcal{O}}\left(\operatorname{Ext}_{\bar{A}}(M, M)^{*}\right) \rightarrow \widehat{\operatorname{Free}}_{\mathcal{O}}\left(\operatorname{Ext}_{\frac{1}{A}}(M, M)^{*}\right)
$$

for $\operatorname{Def}_{M}^{A}$. If $\mathrm{o}_{A}^{A}$ composed with the natural map $\widehat{\mathrm{Free}}_{\mathcal{O}}\left(\operatorname{Ext}_{A} \frac{1}{A}(M, M)^{*}\right) \rightarrow T^{1}$ is trivial, one can choose $\left(\mathrm{o}^{A}, \mathrm{o}^{J}\right)$ for $\operatorname{Def}_{M}^{(A, J)}$ such that $\mathrm{o}^{A}$ is trivial. However, even if $\mathrm{o}^{A}$ in $\left(\mathrm{o}^{A}, \mathrm{o}^{J}\right)$ is trivial, $\mathrm{O}_{A}^{A}$ continued to $T^{1}$ may be far from trivial as Example 5 shows. There is no way one can find $\mathrm{o}^{A}$ "first" and then find $\mathrm{o}^{J}$, as this has no meaning. It is not clear to the author whether $\mathrm{o}^{A}$ in the pair $\left(\mathrm{o}^{A}, \mathrm{o}^{J}\right)$ and the locus it defines has any interesting interpretation.

Proof. Suppose $\left(\mathrm{o}^{A}, \mathrm{o}^{J}\right)$ is given, and assume first that $\operatorname{dim}_{k} \mathrm{H}^{1}<\infty$. By Proposition 2 and Lemma 4 we have a "short exact sequence" $T_{A}^{2} \hookrightarrow T_{B}^{2} \rightarrow T_{J}^{2}$, the last map has a section and if $T_{A}^{2}=\mathcal{O}\left[y_{1}, y_{2}, \ldots\right]$, $T_{J}^{2}=\mathcal{O}\left[z_{1}, z_{2}, \ldots\right]$, we let the union $\left\{y_{i}\right\} \cup\left\{z_{j}\right\}$ also denote the "generators" in $T_{B}^{2}$. We want to define $\mathrm{o}^{B}$. While we let $\mathrm{o}^{B}\left(y_{i}\right):=\mathrm{o}^{A}\left(y_{i}\right)=f_{i} \in T^{1}$ which is OK by Lemma 4, we find $\mathrm{o}^{B}\left(z_{j}\right)=h_{j} \in T^{1}$ by induction. We use notation as in the proof of Theorem 2, except that $f_{i}^{n}, g_{j}^{n}$ and $h_{j}^{n}$ are the images of $f_{i}, g_{j}$ and $h_{j}$ in $T_{n}^{1}$. By Theorem 4, $\mathrm{o}_{B}\left(\pi_{n}^{A}, M_{n-1}\right)=$ $\sum y_{i}^{*} \otimes \bar{f}_{i}^{n}+\sum z_{j}^{*} \otimes \bar{h}_{j}^{n} \in \mathrm{H}_{B}^{2} \otimes_{k} I_{n}^{A}$ maps to $o_{J}\left(\pi_{n}^{J}, M_{n-1}\right)=\sum z_{j}^{*} \otimes \bar{g}_{j}^{n} \in \mathrm{H}_{J}^{2} \otimes_{k} I_{n}^{J}$ along $I_{n}^{A} \rightarrow I_{n}^{J}$. Since $\operatorname{ker}\left(I_{n}^{A} \rightarrow I_{n}^{J}\right)=\left(\bar{f}_{i}^{n}\right)$ we get $\bar{h}_{j}^{n}=\bar{g}_{j}^{n}+\sum \bar{a}_{j i} \bar{f}_{i}^{n}$ with $\bar{a}_{j i} \in k$. By naturality of the obstruction classes, $\bar{h}_{j}^{n}=\bar{g}_{j}^{n}+\sum \bar{a}_{j i} \bar{f}_{i}^{n}$ maps to $\bar{h}_{j}^{n-1}=\bar{g}_{j}^{n-1}+\sum \bar{a}_{j i} \bar{f}_{i}^{n-1}$ under the natural map $G_{n}^{A} \rightarrow G_{n-1}^{A}$. We may assume that the $\bar{f}_{i}^{n-1}$ in the latter sum are $k$-linearly independent. By induction we have chosen $a_{j i}^{(n-1)} \in \mathcal{O} / \mathfrak{m}^{n-1}$ lifting $\bar{a}_{j i}$ and defined $\mathrm{o}_{n-1}^{B}\left(z_{j}\right)=h_{j}^{n-1}=g_{j}^{n-1}+\sum a_{j i}^{(n-1)} f_{i}^{n-1}$ in $T_{n-1}^{1}$. Choose liftings $a_{j i}^{(n)} \in \mathcal{O} / \mathfrak{m}^{n}$ of $\bar{a}_{j i}^{(n-1)}$ and of the "new" coefficients $\bar{a}_{j i}$, i.e. if $a_{j i}^{(n-1)}=0$ (but only such that the $\bar{f}_{i}^{n}$ in the sum $\bar{h}_{j}^{n}=\bar{g}_{j}^{n}+\sum \bar{a}_{j i} \bar{f}_{i}^{n}$ are linearly independent). Put $\mathrm{o}_{n}^{B}\left(z_{j}\right)=h_{j}^{n}=g_{j}^{n}+\sum a_{j i}^{(n)} f_{i}^{n}$ in $T_{n}^{1}$. In the limit we get $\mathrm{o}^{B}\left(z_{j}\right)=h_{j}=g_{j}+\sum a_{j i} f_{i}$, where $a_{j i}=\varliminf_{\longleftarrow}\left\{a_{j i}^{(n)}\right\} \in \mathcal{O} \subseteq T^{1}$. This is stronger than our claim.

For the general case, choose a sequence of finite-dimensional $k$-vector spaces $V_{1} \subset$ $V_{2} \subset \ldots \subset \mathrm{H}^{1}$ with $\bigcup V_{s}=\mathrm{H}^{1}$ as in the proof of Theorem 2. If $\mathrm{o}^{B}\left({ }_{s+1} \pi_{n}^{A}, M_{n-1}\right)=$ $\sum y_{i}^{*} \otimes_{s+1} \bar{f}_{i}^{n}+\sum z_{j}^{*} \otimes_{s+1} \bar{h}_{j}^{n}$, then ${ }_{s+1} \bar{h}_{j}^{n}={ }_{s+1} \bar{g}_{j}^{n}+\sum \bar{a}_{j i}\left({ }_{s+1} \bar{f}_{i}^{n}\right)$, and if $\bar{f}_{i}^{n}=0$, choose liftings $a_{j i}^{(n)} \in \mathcal{O} / \mathfrak{m}^{n}$ of $a_{j i}^{(n-1)}$ and (if also ${ }_{s+1} \bar{f}_{i}^{n-1}=0$ ) of $\bar{a}_{j i}$ and put ${ }_{s+1} \mathrm{O}_{n}^{B}\left(z_{j}\right)={ }_{s+1} h_{j}^{n}={ }_{s+1} g_{j}^{n}+\sum a_{j i}^{(n)}\left({ }_{s+1} f_{i}^{n}\right)$; the other $a_{j i}^{(n)}$ are already chosen by induction on $s$. Put $\mathrm{o}^{B}\left(z_{j}\right)=\lim _{\mathrm{I}}\left\{\mathrm{O}_{n}^{B}\left(z_{j}\right)\right\}$. It is well defined.

Given $\mathrm{o}^{B}$, let $\mathrm{o}^{A}\left(y_{i}\right):=\mathrm{o}^{B}\left(y_{i}\right)$ and let $\mathrm{o}^{J}\left(z_{j}\right):=\mathrm{o}^{B}\left(z_{j}\right)$. Then $\left(\mathrm{o}^{A}, \mathrm{o}^{J}\right)$ are obstruction maps for the two obstructions $\mathrm{o}_{A}$ and $\mathrm{o}_{J}$. This follows from Theorem 4 and Lemma 4

\section{The Change of Rings SPECTRAL SEQUence}

The spectral sequence connects the $A$ - and the $B$-cohomology and also provides a framework for describing relations between the various obstruction classes. In the 
following we give detailed descriptions of the maps $\alpha, d_{2}$ and $\gamma$ by representations in the Yoneda complex.

Lemma 2. Let $A \rightarrow B$ be a ring homomorphism and let $N, M$ be an $A$ - and a $B$ module, respectively. Then there is a first quadrant cohomological spectral sequence

$$
\mathrm{E}_{2}^{p q}=\operatorname{Ext}_{B}^{p}\left(M, \operatorname{Ext}_{A}^{q}(B, N)\right) \Rightarrow \operatorname{Ext}_{A}^{*}(M, N) .
$$

In particular there is a canonical 5-term exact sequence which, in the case $B=A / J$ and $N$ is a B-module as A-module, becomes

$$
\begin{aligned}
& 0 \longrightarrow \operatorname{Ext}_{B}^{1}(M, N) \longrightarrow \operatorname{Ext}_{A}^{1}(M, N) \stackrel{\alpha}{\longrightarrow} \operatorname{Hom}_{A}\left(J, \operatorname{Hom}_{A}(M, N)\right) \\
& \stackrel{d_{2}}{\longrightarrow} \operatorname{Ext}_{B}^{2}(M, N) \stackrel{\gamma}{\longrightarrow} \operatorname{Ext}_{A}^{2}(M, N)
\end{aligned}
$$

Proof. Let $G=G . \rightarrow M$ be a $B$-projective resolution of $M$ and let $N \hookrightarrow I^{\cdot}=I$ be an $A$-injective resolution of $N$. Then the $I I$-filtration of $\operatorname{Hom}_{B}\left(G, \operatorname{Hom}_{A}(B, I)\right)$ gives a spectral sequence which collapses at stage 2 to the total cohomology. The spectral sequence obtained from the $I$-filtration gives the $\mathrm{E}_{2}$-terms. The 5-term exact sequence is the standard one with $\mathrm{E}_{2}^{01} \cong \operatorname{Hom}_{A}\left(J, \operatorname{Hom}_{A}(M, N)\right)$.

Let $\varepsilon:(F, d) \rightarrow M$ be an $A$-free resolution of $M$, and let $E \rightarrow B$ be an $A$-free resolution of $B ; \ldots E_{2} \rightarrow E_{1} \rightarrow A \rightarrow B$. Recall the definition of $m: E \otimes_{A} F \rightarrow F$ before Proposition 1 We change the notation by $s:=m_{\geqslant 1}: E_{\geqslant 1} \otimes F \rightarrow F$, where $E_{\geqslant 1}=\left[\ldots \rightarrow E_{2} \rightarrow E_{1}\right][+1]$, and let $m_{E_{1}}: E_{1} \otimes F \rightarrow F$ be the multiplication with $J=\left(f_{1}, \ldots, f_{r}\right)$ on $F$-map pulled back along $E_{1} \rightarrow J$. Then $\partial(s)$ equals $m_{E_{1}}$ when restricted to $E_{1} \otimes F$ and is zero elsewhere. Hence the map $\partial_{J}$ is described simply as induced by the pullback along $s: E_{1} \otimes F_{0} \rightarrow F_{1}$, (the $m_{1}$ in (4)).

Proposition 2. If $M$ and $N$ are $B$-modules as $A$-modules, then

$$
\partial_{J}: \operatorname{Ext}_{A}^{1}(M, N) \longrightarrow \operatorname{Hom}_{A}\left(J, \operatorname{Hom}_{A}(M, N)\right)
$$

given in Definition 1 is the edge map $\alpha$ in the change of rings spectral sequence in Lemma 圆 In particular there are canonical isomorphisms

$$
\operatorname{ker} \partial_{J} \cong \operatorname{Ext}_{B}^{1}(M, N) \quad \text { and } \quad \text { coker } \partial_{J} \cong \operatorname{im} d_{2} \subseteq \operatorname{Ext}_{B}^{2}(M, N),
$$

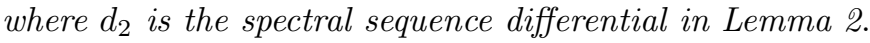

Proof. Let $\xi \in \operatorname{Hom}_{A}\left(F_{1}, N\right)$ be a cocycle representing the class $[\xi] \in \operatorname{Ext}_{A}^{1}(M, N)$. Then there is a $\rho \in \operatorname{Hom}_{A}\left(F_{0}, I^{0}\right)$ extending $\iota \xi$, where $\iota: M \hookrightarrow I^{0}$ is the coaugmentation map. There is also a $\tau \in \operatorname{Hom}_{A}\left(M, I^{1}\right)$ extending $d^{0} \rho$, clearly $[\tau]=$ $[\xi]$. From $\rho d_{1}=\iota \xi$ we get $\rho m_{E_{1}}=\iota \xi s$. The map $\iota \xi s$ represents $\partial_{J}([\xi])$. If $\bar{\varepsilon}=\varepsilon \otimes_{A} B$ one is left to prove that the connecting $\operatorname{Hom}_{B}\left(M, \operatorname{Ext}_{A}^{1}(B, M)\right) \stackrel{\simeq}{\longrightarrow}$ $\operatorname{Hom}_{A}\left(J \otimes_{A} M, N\right) \cong \operatorname{Hom}_{A}\left(J, \operatorname{Hom}_{A}(M, N)\right)$ is represented by taking $\tau \bar{\varepsilon}$ to $\rho m_{E_{1}}$. Applying $\operatorname{Hom}_{A}\left(F_{0}, \operatorname{Hom}_{A}\left(-, I^{*}\right)\right)$ to the short exact sequence $0 \rightarrow J \rightarrow A \rightarrow$ $B \rightarrow 0$ gives an exact sequence of complexes. Observe $\operatorname{Hom}_{A}\left(F_{0}, \operatorname{Hom}_{A}\left(B, I^{\cdot}\right)\right) \cong$ $\operatorname{Hom}_{A}\left(\bar{F}_{0}, I^{\cdot}\right)$ and

$$
\operatorname{Hom}_{A}\left(F_{0}, \operatorname{Hom}_{A}\left(J, I^{\cdot}\right)\right) \cong \operatorname{Hom}_{A}\left(J \otimes F_{0}, I^{\cdot}\right) \hookrightarrow \operatorname{Hom}_{A}\left(E_{1} \otimes F_{0}, I^{\cdot}\right) .
$$


Hence

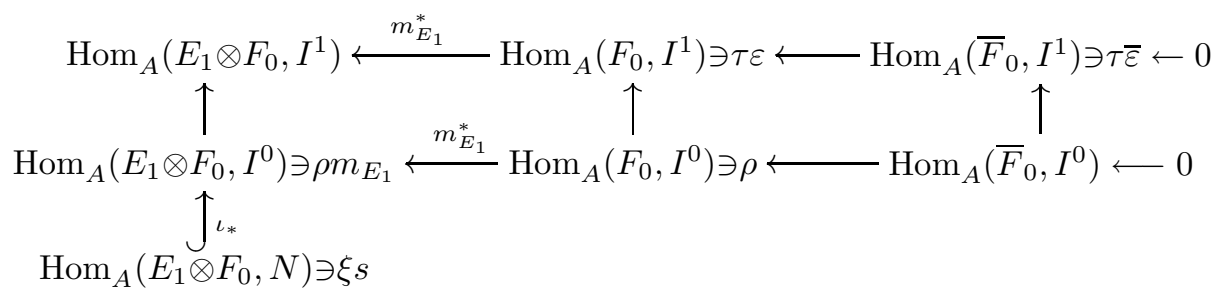

Remark 10. If $A$ is an $\mathcal{O}$-algebra for any commutative $\operatorname{ring} \mathcal{O}$, there is a restriction of derivations map

$$
D \in \operatorname{Der}_{\mathcal{O}}\left(A, \operatorname{Hom}_{\mathcal{O}}(M, N)\right) \longrightarrow \operatorname{Hom}_{A}\left(J, \operatorname{Hom}_{A}(M, N)\right) \ni D_{\mid J}
$$

which one checks is well defined if $M$ and $N$ are annihilated by $J$. The inner derivations map to zero and if $A$ is $\mathcal{O}$-flat we have

$$
\operatorname{Der}_{\mathcal{O}}(A, W) /(\text { inner derivations }) \cong \operatorname{HH}^{1}(A ; W) \cong \operatorname{Ext}_{A}^{1}(M, N),
$$

where $\operatorname{HH}^{*}(A ; W)$ is the Hochschild cohomology with values in the $A$-bimodule $W=\operatorname{Hom}_{\mathcal{O}}(M, N)$. Via this identification our $\partial_{J}$ equals the restriction of derivations map. This is proved by constructing a specific lifting of the multiplication of $J$-map on a non-reduced bar complex. Indeed let $s: E_{1} \otimes_{A} A \otimes_{\mathcal{O}} M \rightarrow A \otimes_{\mathcal{O}} A \otimes_{\mathcal{O}} M$ be defined by $s\left(e_{i} \otimes 1 \otimes m\right)=1 \otimes f_{i} \otimes m$, where $J=\left(f_{1}, \ldots, f_{r}\right)$ and $E_{1}=\bigoplus_{i=1}^{i=r} A e_{i}$. Then $\partial_{J}=s^{*}$. We are indebted to Prof. O. A. Laudal for suggesting this interpretation of $\partial_{J}$ at an early stage, which again led to the above change of rings spectral sequence.

Example 5. Let $P$ be a regular local complete Noetherian $k$-algebra, and let $J_{A}$ and $J_{B}$ be ideals in $P$ with $0 \neq J_{A} \subseteq \mathfrak{m} J_{B}$ and $J_{B} \subseteq \mathfrak{m}^{2}$. Let $A=P / J_{A}$, $B=P / J_{B}, \mathcal{O}=k$ and $M=k$ which is assumed to be the residue field of $B$. Then $\operatorname{Def}_{M}^{X}$ is pro-represented by $X$ for $X=P, A$ and $B$; see Example 4 . In particular the ideal $J_{A}$ is given by the image of the maximal ideal under the obstruction map $\mathrm{o}^{A}$. However, if $\bar{J}:=J_{B} \cdot A \subseteq A$, then $\mathrm{o}^{A}$ in the pair of obstruction maps $\left(\mathrm{o}^{A}, \mathrm{o}^{\bar{J}}\right)$ is trivial. The reason for this can be seen from the obstruction calculus. Since $o^{J}$ can potentially generate $J_{B}$ as obstruction ideal and $\mathrm{o}^{A}$ only generates $J_{A} \subseteq \mathfrak{m} J_{B}$, the $\mathrm{o}_{A}$-obstruction will be one or more "steps" behind $\mathrm{o}_{\bar{J}}$, the latter thus takes care of all the obstruction. This phenomenon can also be deduced from the 5-term exact sequence. For transparency assume $J_{A}$ and $J_{B}$ are generated by regular sequences of length $a$ and $b$. $A$ - and $B$-free minimal resolutions of $k$ may be produced from the $P$-free Koszul resolution of $k$ beginning with the 3-presentation in Lemma 3 below (with $E_{2}^{\prime}=0$ ). Then the 5 -term exact sequence is

$$
0 \longrightarrow \mathfrak{m}_{B} / \mathfrak{m}_{B}^{2} \stackrel{\simeq}{\longrightarrow} \mathfrak{m}_{A} / \mathfrak{m}_{A}^{2 *} \stackrel{0}{\longrightarrow}(\bar{J} \otimes k)^{*} \stackrel{d_{2}}{\longrightarrow} k^{b} \oplus k^{\left(\begin{array}{c}
n \\
2
\end{array}\right)} \longrightarrow k^{a} \oplus k^{\left(\begin{array}{c}
n \\
2
\end{array}\right)}
$$

where $n=\operatorname{dim}_{\text {Krull }} P$. The spectral sequence differential $d_{2}$ is injective, and gives the isomorphism $(\bar{J} \otimes k)^{*} \cong k^{b}$. This in fact also proves that $\mathrm{o}_{B}$ is confined to $k^{b}$ and thus maps to zero in $\operatorname{Ext}_{A}^{2}(k, k)$. Hence $\mathrm{o}^{A}$ in the pair $\left(\mathrm{o}^{A}, \mathrm{o}^{\frac{J}{J}}\right)$ has to be trivial; see Lemma 4. The $o_{A}$ for $\operatorname{Def}_{k}^{A}$ is for the same reason confined to $k^{a}$, but far from trivial. Remark that this is not in contradiction to Lemma 4 If, by changing the assumptions, some elements in $J_{A}$ are non-zero in $J_{B} / \mathfrak{m} J_{B}$, they will produce 
identifications between corresponding subspaces of $k^{b}$ and $k^{a}$ and hence some of $\mathrm{o}^{A}$ will be induced from $\mathrm{O}^{A}$ in $\left(\mathrm{o}^{A}, \mathrm{o}^{\bar{J}}\right)$. In fact the isomorphism of the "Koszul-part" of the Ext ${ }^{2}$ 's may be explained similarly if we for a moment consider the noncommutative deformation functors, i.e. where the local Artinian rings are allowed to be non-commutative. Then $\operatorname{Def}_{k}^{B}$ is in fact still pro-represented by the commutative ring $B$; see Example 4 The $\left(\begin{array}{c}n \\ 2\end{array}\right)$-part takes care of the commutators, which are given as cup products, and hence appear simultaneously in the obstruction calculus for both deformation functors.

Assume $d_{2}: E_{2} \rightarrow E_{1}$ is given as the Koszul differential $d_{K}$ plus a map $d_{E}^{\prime}$ : $E_{2}^{\prime} \rightarrow E_{1} \cong A^{r}$ and let $s^{\prime}$ be the restriction of $s: E_{2} \otimes F_{0} \rightarrow F_{2}$ to $E_{2}^{\prime} \otimes F_{0}$.

Lemma 3. The following maps of A-free modules give a B-free 3-presentation of $M$ after tensoring with $B$ :

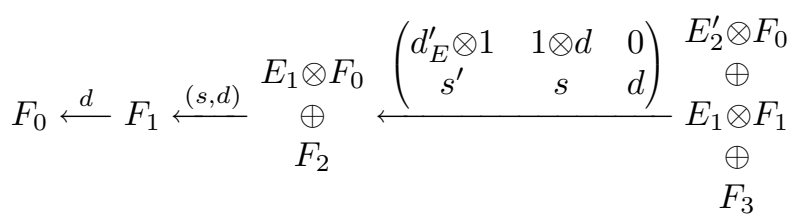

Remark 11. The above assumption about $d_{2}: E_{2} \rightarrow E_{1}$ is no limitation; we can always produce such resolutions of $B$. The point is that if $J=\left(f_{1}, \ldots, f_{r}\right)$ is a regular sequence, then $\mathrm{H}_{1}\left(K\left(f_{1}, \ldots, f_{r}\right)\right)=0$ where $K$ is the Koszul complex, $E_{2}^{\prime}=0$ and our 3-presentation is the beginning of a construction of Eisenbud which gives a $B$-free resolution from an $A$-free one; see [4, Theorem 7.1]. In general this resolution is not minimal. Using $E_{2}$ instead of $E_{2}^{\prime}$, it is not hard to prove the result by moving elements around.

Proof. Since the 3-presentation (with $E_{2}$ instead of $E_{2}^{\prime}$ ) is the beginning of the mapping cone $K(s)$ of $B \otimes s: B \otimes E \geqslant 1 \otimes F \rightarrow B \otimes F[+1]$ shifted minus one, and the composition $E_{\geqslant 1} \otimes F \rightarrow B \otimes E_{\geqslant 1} \otimes F \rightarrow B \otimes F[+1]$ is a quasi-isomorphism inducing the inverse connecting $\operatorname{Tor}_{*}^{A}(J, M) \stackrel{\simeq}{\longrightarrow} \operatorname{Tor}_{*+1}^{A}(B, M)$, the map $\mathrm{H}_{*}(B \otimes s)$ is surjective. Thus $\mathrm{H}_{i} K(s)=0$ for $i=0,1$ and hence our complex is exact in degree 1 and 2 .

Let $(\mathrm{id}[2], 0)$ be the obvious map $F_{0} \otimes E_{1}[-1] \oplus F_{2} \rightarrow F_{0} \otimes E_{1}[1]$ tensored down to $B$. The map induces a 2 -cocycle in a $B$-free Yoneda complex which calculates $\operatorname{Ext}_{B}^{*}\left(M, M \otimes_{B} J / J^{2}\right)$, where the resolution of $M$ begins as in Lemma 3 and the resolution of $M \otimes_{B} J / J^{2}$ begins with $B \otimes_{A}\left(F_{0} \otimes E_{1}\right)$. In fact this cocycle represents o $:=\mathrm{o}\left(A / J^{2}\right) \in \operatorname{Ext}_{B}^{2}\left(M, M \otimes_{B} J / J^{2}\right)$ which is the obstruction for lifting $M$ to $A / J^{2}$ as in Definition [4 This element induces via the Yoneda cup product $\operatorname{Hom}_{B}\left(M \otimes_{B} J / J^{2}, N\right) \times \operatorname{Ext}_{B}^{2}\left(M, M \otimes_{B} J / J^{2}\right) \stackrel{\cup}{\longrightarrow} \operatorname{Ext}_{B}^{2}(M, N)$ a map

$$
-\cup \mathrm{o}: \operatorname{Hom}_{B}\left(M \otimes_{B} J / J^{2}, N\right) \longrightarrow \operatorname{Ext}_{B}^{2}(M, N) \text {. }
$$

Via the natural isomorphism $\operatorname{Hom}_{B}\left(M \otimes_{B} J / J^{2}, N\right) \stackrel{\simeq}{\longrightarrow} \operatorname{Hom}_{B}\left(M, \operatorname{Ext}_{A}^{1}(B, N)\right)$ we have:

Proposition 3. Assume both $M$ and $N$ are $B=A / J$-modules as $A$-modules. Then the differential in the change of rings spectral sequence of Lemma $Q$

$$
d_{2}: \operatorname{Hom}_{B}\left(M, \operatorname{Ext}_{A}^{1}(B, N)\right) \longrightarrow \operatorname{Ext}_{B}^{2}(M, N)
$$


is induced by cupping with the obstruction $\mathrm{o}\left(A / J^{2}\right) \in \operatorname{Ext}_{B}^{2}\left(M, M \otimes_{B} J / J^{2}\right)$ as in (10) and $\mathrm{o}\left(A / J^{2}\right)$ is induced by the cocycle (id[2],0) in the Yoneda complex of B-free resolutions. In particular the obstruction is canonically given as

$$
\mathrm{o}\left(A / J^{2}\right)=d_{2}\left(\mathrm{id}_{M \otimes J / J^{2}}\right) .
$$

Remark 12. This result is only marginally different from L. Illusie's Propositions 3.1.5 and 3.1.13 combined [10, Chap. IV], in the case of rings (Illusie works with rings over a topos). In his formulation $J^{2}=0\left(\operatorname{but}_{\operatorname{Ext}_{A}^{1}}^{1}(M, N) \cong \operatorname{Ext}_{A / J^{2}}^{1}(M, N)\right)$, and his spectral sequence is $\operatorname{Ext}_{B}^{p}\left(\operatorname{Tor}_{q}^{A / J^{2}}(M, B), N\right) \Rightarrow \operatorname{Ext}_{A / J^{2}}^{*}(M, N)$. Illusie's proof depends on the cotangent complex of graded algebras and gives representations in the derived category. We are interested in explicit calculations of the generalised Massey products and the formally versal formal family, and our proof, which gives simple representations of the map and the class in the Yoneda complex, is therefore better suited to our needs.

Remark 13. If $J$ defines a locally complete intersection, i.e. $J / J^{2}$ is $B$-projective of finite rank, it is not hard to extend the result to all the $d_{2}$-differentials. We have

$$
\mathrm{E}_{2}^{p q}=\operatorname{Ext}_{B}^{p}\left(M, \operatorname{Ext}_{A}^{q}(B, N)\right) \cong \operatorname{Ext}_{B}^{p}(M, N) \otimes \bigwedge^{q} J / J^{2^{*}}
$$

and $\psi \in \operatorname{End}_{B}\left(J / J^{2}\right)$ acts on $f_{1} \wedge \ldots \wedge f_{q} \in \wedge J / J^{2^{*}}$ by $\psi \cdot f_{1} \wedge \ldots \wedge f_{q}=\sum f_{1} \wedge$ $\ldots \wedge \psi^{*}\left(f_{i}\right) \wedge \ldots \wedge f_{q}$ while $J / J^{2}$ acts as graded derivations. Combining this with the cup product, any $\xi \in \mathrm{E}_{2}^{p q}=\operatorname{Ext}_{B}^{p}\left(M, \operatorname{Ext}_{A}^{q}(B, N)\right)$ defines a natural, vertical map by "multiplication" in the diagram:

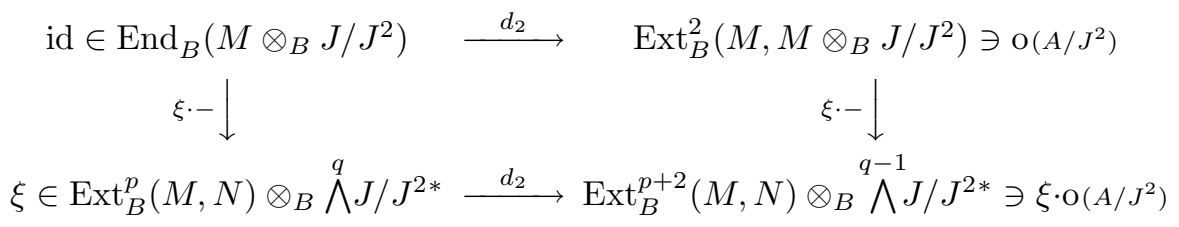

The diagram commutes since $d_{2}$ is natural for natural products on the cohomology and hence if $\mathrm{o}\left(A / J^{2}\right)=\sum \mathrm{o}_{i} \otimes x_{i}$ and $\xi=\zeta \otimes f$, then $d_{2}(\xi)=\sum\left(\zeta \cup \mathrm{o}_{i}\right) \otimes f \cdot x_{i}$.

Proof of Proposition 3. The pullback of endomorphisms by (id[2],0) induces a map

$$
\operatorname{End}_{B}\left(M \otimes J / J^{2}\right) \longrightarrow \operatorname{Ext}_{B}^{2}\left(M, M \otimes J / J^{2}\right)
$$

which possibly depends on the resolution chosen, etc. We show that the image of the identity is the obstruction $\mathrm{O}\left(A / J^{2}\right)$ which follows since $\mathrm{O}\left(A / J^{2}\right)$ by construction (see the proof of Theorem 1) is induced from the square of a lifting to $A / J^{2}$ of the $B$-differential $d^{B}$ in a $B$-free resolution $\left(F^{B}, d^{B}\right)$ of $M$ : Recall the notation before Proposition 2. By Lemma 3, $d^{B}$ may be chosen with $d_{1}^{B}=d_{1} \otimes_{A} B, d_{2}^{B}=$ $\left(s, d_{2}\right) \otimes_{A} B$ hence $\tilde{d_{1}^{B}} \circ \tilde{d}_{2}^{B}=d_{1} \circ\left(s, d_{2}\right) \otimes_{A} A / J^{2}=\left(m_{E_{1}}, 0\right) \otimes_{A} A / J^{2}$ which lifts to $(\mathrm{id}[2], 0)$ via $m_{E_{1}}: F_{0} \otimes E_{1} \rightarrow F_{0}$. Since we have such a nice representation of $\mathrm{o}\left(A / J^{2}\right)$ in the Yoneda complex, the idea of the proof is to take a class $[\xi] \epsilon$ $\operatorname{Hom}_{B}\left(M, \operatorname{Ext}_{A}^{1}(B, N)\right) \cong \operatorname{Hom}_{B}\left(M \otimes J / J^{2}, N\right)$ represented by a cocycle $\xi=\left(\xi_{i}\right)$ in the Yoneda complex: $\xi_{0} \in \operatorname{Hom}_{B}\left(\bar{F}_{0} \otimes \bar{E}_{1}, \bar{F}_{0}^{\prime}\right)$, where $\left(F^{\prime}, d^{\prime}\right)$ is an $A$-free resolution of $N$ and $\bar{F}=F \otimes_{A} B$, etc. Move it to a representative for the same class in $\mathrm{E}^{01}=\operatorname{Hom}_{B}\left(F_{0}^{B}, \operatorname{Hom}_{A}\left(B, I^{1}\right)\right)$, where $F_{0}^{B}=\bar{F}_{0}$ and $N \hookrightarrow I$ is an $A$-injective resolution of $N$, and calculate $d_{2}$ by moving this new representative along the "stairs" in the double complex $\operatorname{Hom}_{B}\left(F^{B}, \operatorname{Hom}_{A}(B, I)\right)$ to a representative for the 
image $d_{2}([\xi])$ in $\mathrm{E}^{20}=\operatorname{Hom}_{B}\left(F_{2}^{B}, \operatorname{Hom}_{A}\left(B, I^{0}\right)\right)\left(\right.$ where $\left.F_{2}^{B}=\bar{F}_{0}[2] \otimes \bar{E}_{1} \oplus \bar{F}_{2}\right)$. Finally we move back to a representative for $d_{2}([\xi])$ in the Yoneda complex and observe that we may take it to be $\left(\xi_{0}[2], 0\right) \in \operatorname{Hom}_{B}\left(\bar{F}_{0}[2] \otimes \bar{E}_{1} \oplus \bar{F}_{2}, \bar{F}_{0}^{\prime}\right)$. Since $\xi_{0} \circ(\operatorname{id}[2], 0)=\left(\xi_{0}[2], 0\right)$, we get $d_{2}([\xi])=[\xi] \cup \mathrm{o}\left(A / J^{2}\right)$. Hence the map [11) induced by the particular form of the (possibly non-minimal) 3-presentation is indeed canonical and equal to $d_{2}$. This is only almost what we do, actually we lift the $B$-representative $\xi_{0}$ from $\operatorname{Hom}_{B}\left(\bar{F}_{0}, \operatorname{Hom}_{B}\left(\bar{E}_{1}, \bar{F}_{0}^{\prime}\right)\right)$ to an $A$-representative in $\operatorname{Hom}_{A}\left(F_{0}, \operatorname{Hom}_{A}\left(E_{1}, F_{0}^{\prime}\right)\right)$ and then do the zigzagging with $A$-representatives. Details are given in [8].

\section{The Formula $\mathrm{o}(J) \cup \mathrm{o}\left(A / J^{2}\right)=\mathrm{O}_{B}$}

Several of our obstruction classes are connected by the $d_{2}$-differential in the following theorem.

Theorem 4. With assumptions as in Theorem 1, assume furthermore that there exists an $A_{R}$-module $M_{R}$ lifting $M$ along $\pi_{A_{R}}=\mathrm{id}_{A} \otimes \pi$ in the diagram

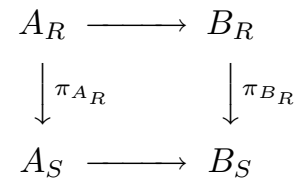

in the sense of Definition 4 in particular $\mathrm{o}_{A_{S}}\left(\pi_{A_{R}}, M\right)=0$. If $\mathrm{O}_{J_{S}}=\mathrm{o}_{J_{S}}\left(\pi_{A_{R}}, M\right)$ is the obstruction for lifting $M$ to $B_{R}$, then, via the natural inclusion $\mathrm{o}_{J_{S}} \in \operatorname{coker} \partial_{J_{S}}$ $\hookrightarrow \operatorname{Ext}_{B_{S}}^{2}\left(M, M \otimes_{S} I\right)$, it satisfies

$$
\mathrm{o}_{J_{S}}=d_{2}\left(\mathrm{o}\left(J_{S}\right)\right)=\mathrm{o}\left(J_{S}\right) \cup \mathrm{o}\left(A_{S} / J_{S}^{2}\right)=\mathrm{o}_{B_{S}},
$$

where $\cup$ is the cup product and $\mathrm{o}\left(J_{S}\right) \in \operatorname{Hom}_{B_{S}}\left(M \otimes_{B_{S}} J_{S} / J_{S}^{2}, M \otimes_{S} I\right)$ is the obstruction for $M_{R}$ to be a $B_{R}$-module as $A_{R}$-module,

$$
\mathrm{o}\left(A_{S} / J_{S}^{2}\right) \in \operatorname{Ext}_{B_{S}}^{2}\left(M, M \otimes_{B_{S}} J_{S} / J_{S}^{2}\right)
$$

is the obstruction for lifting $M$ to $A_{S} / J_{S}^{2}, \mathrm{o}_{B_{S}} \in \operatorname{Ext}_{B_{S}}^{2}\left(M, M \otimes_{S} I\right)$ is the obstruction for lifting $M$ to $B_{R}$ and

$$
d_{2}: \operatorname{Hom}_{B_{S}}\left(M \otimes_{B_{S}} J_{S} / J_{S}^{2}, M \otimes_{S} I\right) \rightarrow \operatorname{Ext}_{B_{S}}^{2}\left(M, M \otimes_{S} I\right)
$$

is the $2^{\text {nd }}$ differential in the change of rings spectral sequence in LemmaQ

Proof. Let $(F, d)$ be an $A_{S}$-free resolution of $M$. Then $\mathrm{o}_{A_{S}}$ is induced by $\tilde{d}_{1} \circ \tilde{d}_{2}$, where $(\tilde{F}, \tilde{d})$ is a lifting of $d$ to maps of $A_{R}$-free modules as explained in the proof of Theorem 1. We choose an $A_{S}$-free $E_{1} \rightarrow J_{S}$ and maps $s$ as in Lemma 3 to produce a $B_{S}$-free 3 -presentation $F^{B_{S}}=F^{B_{S}}(s)$ of $M$. We observe the bottom row as $B_{S} \otimes d$ and that the edge map $\operatorname{Ext}_{B_{S}}^{2}(M,-) \rightarrow \operatorname{Ext}_{A_{S}}^{2}(M,-)$ in the change of rings spectral sequence is induced by the map $F \rightarrow F^{B_{S}}(s)$, where $F$ maps to $B_{S} \otimes F$; see Lemma 4. The obstruction $\mathrm{o}_{B_{S}}$ is induced by $\tilde{d}_{1}^{B_{S}} \circ \tilde{d}_{2}^{B_{S}}$, where $\tilde{d}^{B_{S}}$ lifts $d^{B_{S}}$ to an $B_{R}$-free lifting of $F^{B_{S}}$, but $d_{1}^{B_{S}}=B_{S} \otimes d_{1}$ and $d_{2}^{B_{S}}=B_{S} \otimes\left(s, d_{2}\right)$, hence

$$
\tilde{d}_{1}^{B_{S}} \circ \tilde{d}_{2}^{B_{S}}=\widetilde{B_{S} \otimes d_{1}} \circ\left(\widetilde{B_{S} \otimes s}, \widetilde{B_{S} \otimes d_{2}}\right)=B_{R} \otimes A_{R}\left(\tilde{d}_{1} \circ \tilde{s}, \tilde{d}_{1} \circ \tilde{d}_{2}\right),
$$

for liftings $\tilde{d}, \tilde{s}$ of $d$ and $s$ to $A_{R}$-free modules. Clearly $\tilde{d}_{1} \circ \tilde{d}_{2}$ induces the same element as $B_{R} \otimes_{A_{R}} \tilde{d}_{1} \circ \tilde{d}_{2}$ in $\operatorname{Ext}_{A_{S}}^{2}\left(M, M \otimes_{S} I\right)$, hence $o_{B_{S}}$ maps to $o_{A_{S}}$ by the edge map. We have assumed $\mathrm{o}_{A_{S}}=0$, indeed we have chosen an $A_{R}$-module $M_{R}$ lifting 
$M$ which corresponds to a choice of liftings $\tilde{d}_{i}, i=1,2$ with $\tilde{d}_{1} \circ \tilde{d}_{2}=0$, hence $\mathrm{O}_{B_{S}}$ is induced by $\tilde{d}_{1}^{B_{S}} \circ \tilde{d}_{2}^{B_{S}}=B_{R} \otimes_{A_{R}}\left(\tilde{d}_{1} \circ \tilde{s}, 0\right)$ and $\tilde{d}_{1} \circ \tilde{s}$ induces the class $\mathrm{O}\left(J_{S}\right)$ (see the proof of Theorem [1). The only thing we lack is a description of the $d_{2}$ differential in terms of our construction. By Proposition $3 d_{2}$ is cupping with the obstruction class $\mathrm{o}\left(A_{S} / J_{S}^{2}\right) \in \operatorname{Ext}_{B_{S}}^{2}\left(M, M \otimes_{B_{S}} J_{S} / J_{S}^{2}\right)$ which is induced by the composition $\hat{d}_{1}^{B_{S}} \circ \hat{d}_{2}^{B_{S}}$ of liftings of the $B_{S^{-}}$-differential $d_{i}^{B_{S}}$ to maps of an $A_{S} / J_{S}^{2}$-free lifting of $F^{B_{S}}$. However we already have a lifting to $A_{S}$, hence we choose $\hat{d}_{1}^{B_{S}}=$ $A_{S} / J_{S}^{2} \otimes d_{1}$ and $\hat{d}_{2}^{B_{S}}=A_{S} / J_{S}^{2} \otimes\left(s_{0}, d_{2}\right)$ and thus $\mathrm{o}\left(A_{S} / J_{S}^{2}\right)$ is induced by $\hat{d}_{1}^{B_{S}} \circ \hat{d}_{2}^{B_{S}}=$ $A_{S} / J_{S}^{2} \otimes\left(d_{1} \circ s, 0\right)$; the first coordinate corresponds to $\operatorname{id}_{M \otimes J_{S} / J_{S}^{2}}$. Hence the class $\mathrm{o}\left(J_{S}\right)$ maps to $\mathrm{O}_{B_{S}}$ under the spectral sequence differential $d_{2}$ :

$$
\mathrm{o}\left(J_{S}\right) \in \operatorname{Hom}_{B_{S}}\left(M \otimes_{B_{S}} J_{S} / J_{S}^{2}, M \otimes_{S} I\right) \stackrel{d_{2}}{\longrightarrow} \operatorname{Ext}_{B_{S}}^{2}\left(M, M \otimes_{S} I\right) \ni \mathrm{o}_{B_{S}}=\left(\mathrm{o}\left(J_{S}\right), 0\right) .
$$

Finally $\mathrm{o}_{J_{S}}$ is the image of $\mathrm{o}\left(J_{S}\right)$ by $d_{2}$ via the canonical isomorphism coker $\partial_{J_{S}} \cong$ im $d_{2}$ in Proposition 2

For the sake of completeness we note

Lemma 4. The edge map

$$
\gamma: \operatorname{Ext}_{B}^{2}(M, N) \longrightarrow \operatorname{Ext}_{A}^{2}(M, N)
$$

in the 5-term exact sequence (9) is induced by any comparison map $F^{A} \rightarrow F^{B}$ of an $A$ - and a $B$-free resolution of $M$. With notation as in Theorem 4 the obstruction class $\mathrm{O}_{B_{S}}$ maps to the obstruction class $\mathrm{O}_{A_{S}}$ under the edge map

$$
\operatorname{Ext}_{B_{S}}^{2}\left(M, M \otimes_{S} I\right) \longrightarrow \operatorname{Ext}_{A_{S}}^{2}\left(M, M \otimes_{S} I\right) .
$$

Proof. Let $N \hookrightarrow I$ be an $A$-injective resolution of $N$ and consider the complex $\operatorname{Hom}_{B}\left(F^{B}, \operatorname{Hom}_{A}(B, I)\right)$ which induces the spectral sequence in Lemma 2 The edge, which is

$$
\mathrm{E}_{2}^{p 0}=\mathrm{H}^{p} \operatorname{Hom}_{B}\left(F^{B}, \operatorname{Hom}_{A}(B, N)\right) \stackrel{i_{*}}{\longrightarrow} \mathrm{H}^{p}=\mathrm{H}^{p} \operatorname{Hom}_{B}\left(F^{B}, \operatorname{Hom}_{A}(B, I)\right)
$$

for $i: N \hookrightarrow I^{0}$, factorises via

$$
\begin{aligned}
\operatorname{Hom}_{B}\left(F^{B}, \operatorname{Hom}_{A}(B, N)\right) \longrightarrow & \operatorname{Hom}_{A}\left(F^{A}, N\right) \stackrel{\sim}{\longrightarrow} \operatorname{Hom}_{A}\left(F^{A}, I\right) \\
& \sim \operatorname{Hom}_{A}(M, I) \cong \operatorname{Hom}_{B}\left(M, \operatorname{Hom}_{A}(B, I)\right) .
\end{aligned}
$$

That $\mathrm{O}_{B_{S}}$ maps to $\mathrm{O}_{A_{S}}$ follows as in the proof of Theorem 4 .

In the situation of Theorem4, the map $\partial_{I}: \operatorname{Ext}_{A_{R}}^{1}\left(M, M \otimes_{S} I\right) \rightarrow \operatorname{End}_{A_{S}}\left(M \otimes_{S} I\right)$ is surjective by Proposition 3 Hence the following result gives a characterisation of the class $\mathrm{O}\left(J_{S}\right)$.

Proposition 4. With assumptions as in Theorem 4.

$$
\mathrm{o}\left(J_{S}\right)=-\partial_{J_{S}}(\xi)
$$

for any extension $\xi \in \operatorname{Ext}_{A_{R}}^{1}\left(M, M \otimes_{S} I\right)$ with $\partial_{I}(\xi)=\mathrm{id} \in \operatorname{End}_{A_{S}}\left(M \otimes_{S} I\right)$.

A proof in the deformation situation is given in [8]; it is easily extended. 


\section{EXPLICIT EXAMPLES OF OBSTRUCTION CALCULATIONS}

Finally we give some examples where we calculate the obstruction classes in Theorem[1] and the obstruction maps in Theorem 2] In the first example we already know the answer by the general Example 4

Example 6. Let $\mathbb{Z}_{2}=\hat{\mathbb{Z}}_{(2)}$ and $B:=\mathbb{Z}_{2}[x] / J$, where $J=(f)$ and $f=2+x^{2}$. Let $M=\bar{B} /(x) \cong \mathbb{F}_{2}$ as a $\bar{B}=B /(2)$-module. We calculate the obstruction polynomial of $\operatorname{Def}_{M}^{B}:$ Art $_{\mathbb{Z}_{2}} \rightarrow$ Sets. Let $A:=\mathbb{Z}_{2}[x], \bar{A}=A /(2)$. Then $M \leftarrow \bar{A} \leftarrow \bar{A}$ is a length $1 \bar{A}$-free resolution of $M$. We have $\operatorname{Ext} \frac{1}{B}(M, M) \cong \operatorname{Ext} \frac{1}{A}(M, M) \cong \mathbb{F}_{2}$. Since $\operatorname{Ext} \frac{2}{A}(M, M)=0$, there is no $\mathrm{o}_{A}$-obstruction. To find the $\mathrm{o}_{J}$-obstruction we start with a factorisation of the multiplication-by- $f$-map, given by $f \equiv x \cdot x \bmod (2)$; $\bar{A} \stackrel{x}{\longleftarrow} \bar{A} \stackrel{x}{\longleftarrow}$. Let $T^{1}=\mathbb{Z}_{2}[u]^{\wedge}$, where the image $\bar{u}$ of $u$ in $\mathfrak{m} / \mathfrak{n}$, where $\mathfrak{n}=\mathfrak{m}^{2}+(2) T^{1}$ corresponds to the $\mathbb{F}_{2}$-dual of $\xi=[-1]=[1] \in \operatorname{Ext} \frac{1}{B}(M, M)$. Then the universal lifting $M_{1}$ of $M$ to the relative Zariski tangent space $\operatorname{Def}_{M}^{B}(k[\varepsilon])$ is given by the factorisation $A \otimes_{\mathbb{Z}_{2}} T_{1}^{1} \stackrel{x-\bar{u}}{\longleftarrow} A \otimes_{\mathbb{Z}_{2}} T_{1}^{1} \stackrel{x+\bar{u}}{\longleftarrow} A \otimes_{\mathbb{Z}_{2}} T_{1}^{1}$ of $f \equiv x^{2} \bmod \left(2, u^{2}\right)$, where $T_{1}^{1}=T^{1} / \mathfrak{n}=\mathbb{Z}_{2}\left[u\left\lceil/\left(2, u^{2}\right)=\mathbb{F}_{2}[u] /\left(u^{2}\right)\right.\right.$. The only obstruction appears when we try to lift this factorisation of $f$ to $T_{2}^{1}=T^{1} / \mathfrak{n} \cdot \mathfrak{m}=\mathbb{Z}_{2}[u] /\left(2^{2}, 2 u, u^{3}\right)$ and it is represented by $(x-u)(x+u)-f=-\left(2+u^{2}\right)$ in $A \otimes_{\mathbb{Z}_{2}} T_{2}^{1}$. In particular the class $\xi \cup \xi=[-1]=[1] \in$ coker $\partial_{\bar{J}} \cong \mathbb{F}_{2}$ is "carrying" the obstruction polynomial $2+u^{2}$. There are no more obstructions.

If instead $f=4+x^{3}$, one obtains the factorisation

$$
A \otimes_{\mathbb{Z}_{2}} T_{2}^{1} \stackrel{x-u}{\longleftarrow} A \otimes_{\mathbb{Z}_{2}} T_{2}^{1} \stackrel{x^{2}+x u+u^{2}}{\longleftarrow} A \otimes_{\mathbb{Z}_{2}} T_{2}^{1}
$$

of $f \equiv x^{3}$ mod $\left(2^{2}, 2 u, u^{3}\right)$ which gives a defining system $\mathcal{B}$ for the $J$-Massey product $\langle\xi, \xi, \xi ; \mathcal{B}\rangle_{J}=[-1] \in$ coker $\partial_{\bar{J}}$ and the obstruction is $(x-u)\left(x^{2}+x u+u^{2}\right)$ $-\left(4+x^{3}\right)=-\left(4+u^{3}\right)$ (or more precisely $\left.[-1] \otimes\left(4+u^{3}\right)\right)$. There are no more obstructions.

Example 7. Let $C_{4}$ be the cyclic group of order four and $\mathbb{Z}_{2}=\hat{\mathbb{Z}}_{(2)}$. Let $x$ be a generator of $C_{4}$ such that the group algebra $B:=\mathbb{Z}_{2} C_{4} \cong \mathbb{Z}_{2}[x] / J$, where $J=(f)$ and $f=x^{4}-1$. Then $\bar{B}=B /(2)$, let $M=\bar{B} /\left(y^{2}\right) \cong \mathbb{F}_{2}[y] /\left(y^{2}\right)$, where $y=x-1$. We give obstruction polynomials defining the hull $H$ of $\operatorname{Def}_{M}^{B}: \operatorname{Art}_{\mathbb{Z}_{2}} \rightarrow$ Sets and indicate how to find them. Let $A:=\mathbb{Z}_{2}[x]$; then $\bar{A}=A /(2)$. The 5 -term exact sequence of the spectral sequence $\operatorname{Ext} \frac{p}{B}\left(M, \operatorname{Ext} \frac{q}{A}(\bar{B}, M)\right) \Rightarrow \operatorname{Ext} \frac{*}{A}(M, M)($ see Lemma 2] is

$$
0 \rightarrow M \stackrel{\simeq}{\longrightarrow} M \stackrel{\partial_{\bar{J}}=0}{\longrightarrow} M \stackrel{\simeq}{\longrightarrow} M \rightarrow 0
$$

Since $\operatorname{pdim}_{\bar{A}}(M)=1$ there is no $A$-obstruction and $\mathrm{O}^{A}$ is trivial. Let $T_{J}^{2}=$ $\mathbb{Z}_{2}\left[z_{0}, z_{1}\right]$, where $\bar{z}_{0}$ and $\bar{z}_{1}$, the images in the relative cotangent space, are $\mathbb{F}_{2^{-}}$ duals to the elements -1 and $-y$ of coker $\partial_{\bar{J}} \cong M$. Let $T^{1}=\mathbb{Z}_{2}[a, b]^{\wedge}$ where $\bar{a}$ and $\bar{b}$ likewise are $\mathbb{F}_{2}$-duals to the elements -1 and $-y$ in ker $\partial_{\bar{J}} \cong M$. Then the obstruction map o $\mathrm{o}^{J}: T_{J}^{2} \rightarrow T^{1}$ may be given as

$$
\begin{aligned}
& \mathrm{o}^{J}\left(z_{0}\right)=a^{2}+6 a+a b^{2}+4 a b, \\
& \mathrm{o}^{J}\left(z_{1}\right)=4+6 b+b^{3}+4 a+2 a b+4 b^{2} .
\end{aligned}
$$

This is a regular sequence, hence $\operatorname{dim}_{\text {Krull }} H=1$. The versal family is the cyclic module $A \hat{\otimes} H /\left(y^{2}-a-y b\right)$, where $A \hat{\otimes} H=\lim _{\longleftarrow}\left\{A \otimes H / \operatorname{im}\left(\mathfrak{n} \cdot \mathfrak{m}_{H}^{i}\right)\right\}, \mathfrak{n}=\mathfrak{m}_{H}^{2}+(2) H$. 
To find the obstruction one deforms the pair $\left(y^{2}, y^{2}\right)$ as a factorisation of $f ; x^{4}-1=$ $y^{2} \cdot y^{2}$. In particular; $\bar{B} \stackrel{\bar{y}^{2}}{\longleftarrow} \bar{B} \stackrel{\bar{y}^{2}}{\longleftarrow} \bar{B}$ gives a $\bar{B}$-free 2 -presentation of $M$. The universal lifting $M_{1}$ of $M$ to the relative Zariski tangent space $\operatorname{Def}_{M}^{B}(k[\varepsilon])$ is given by the factorisation $x^{4}-1=\left(y^{2}-(a+y b)\right) \cdot\left(y^{2}+(a+y b)\right) \bmod \mathfrak{n}$. The obstructions are created as one lifts and expands the factorisation as to be valid over $A \otimes H / \mathfrak{n m}_{H}^{i}$ successively for all $i$. Indeed we get $x^{4}-1=\left(y^{2}-a-y b\right) \cdot\left(y^{2}+a+y b+6+b^{2}+4 y+4 b\right)$ in $A \hat{\otimes} H$.

In the last example we shall see (even clearer) how the change of rings formalism is instrumental both in estimating and calculating the obstruction. The 5-term exact sequence and the $A$-free Koszul resolution immediately imply that we can have at maximum two obstruction polynomials even though $\operatorname{dim}_{k} \operatorname{Ext}_{B}^{2}(M, M)=4$. Moreover we only have to lift a "generalised matrix factorisation" (see [8]), defined over the regular ring $A$, to give defining systems for the Massey products which calculate the obstruction of $\operatorname{Def}_{M}^{B}$, and hence we avoid the relations in $B$ in the calculus.

Example 8. Let $A=k[x, y], f=x^{m+1}+y^{n+1}, B=A /(f), M=B /\left(y, x^{2}\right)$ as a $B$-module and assume $m \geqslant 3, m \equiv 1 \bmod 2$, and $n \geqslant 1$ (the case $m \equiv 0 \bmod 2$ is similar). We give the obstruction polynomials of $\operatorname{Def}_{M}^{B}: \operatorname{Art}_{k} \rightarrow$ Sets and indicate how to find them. The 5 -term exact sequence is

$$
0 \rightarrow M^{\oplus 2} \stackrel{\simeq}{\longrightarrow} M^{\oplus 2} \stackrel{0}{\rightarrow} M \stackrel{(\mathrm{id}, 0)}{\longrightarrow} M \oplus M \stackrel{(0, \mathrm{id})}{\longrightarrow} M \rightarrow 0
$$

and in particular the Zariski tangent space of the hull $H$ of $\operatorname{Def}_{M}^{B}$ is 4-dimensional as a $k$-vector space; $\operatorname{Ext}_{B}^{1}(M, M) \cong M^{\oplus 2} \cong k^{\oplus 4}$. The Koszul complex $(K, d)$ of $\left(y, x^{2}\right)$ gives an $A$-free resolution of $M$, the Koszul complex is functorial in the sequence (i.e. in the first differential) so that a perturbation of $\left(y, x^{2}\right)$ induce a perturbation $(\widetilde{K}, \tilde{d})$ of the complex, in particular $\tilde{d}^{2}=0$, and hence there is no $A$-obstruction and the obstruction map $\mathrm{O}^{A}$ is trivial. The $d_{2}$-map (9) is injective and the obstruction space coker $\partial_{J} \cong M \cong k^{\oplus 2}$ ( $k$-linearly), where $J=(f)$. Let $T_{J}^{2}=k\left[\left[z_{0}, z_{1}\right]\right]$, where the images $\bar{z}_{0}$ and $\bar{z}_{1}$ in the cotangent space $\mathfrak{m} / \mathfrak{m}^{2}$ are the $k$-dual elements to -1 and $-x$, which give a $k$-basis for coker $\partial_{J}$. Let $T^{1}=k[[a, b, c, d]]$, where $\bar{a}$ and $\bar{b}$ in $\mathfrak{m} / \mathfrak{m}^{2}$ are $k$-duals to -1 and $-x$ in $M$ and likewise for $\bar{c}$ and $\bar{d}$. Let $l=\frac{m-1}{2}$. Then the obstruction map o $\mathrm{o}^{J} T_{J}^{2} \rightarrow T^{1}$ may be given as

$$
\begin{aligned}
\mathrm{o}^{J}\left(z_{0}\right) & =\sum_{i=0}^{n} \sum_{j=0}^{\min (i, n-i)}\left(\begin{array}{c}
i \\
j
\end{array}\right)\left(\begin{array}{c}
n \\
i+j
\end{array}\right) a^{n-i-j} b^{i+j} c^{j+1} d^{i-j} \\
& +\sum_{i=0}^{l}\left(\sum_{j=0}^{\min (i, l-i)}\left(\begin{array}{c}
i \\
j
\end{array}\right)\left(\begin{array}{c}
l \\
i+j
\end{array}\right)\right) c^{l+1-i} d^{2 i}, \\
\mathrm{o}^{J}\left(z_{1}\right) & =\sum_{i=0}^{n} \sum_{j=0}^{\min (i+1, n-i)}\left(\begin{array}{c}
i+1 \\
j
\end{array}\right)\left(\begin{array}{c}
n \\
i+j
\end{array}\right) a^{n-i-j} b^{i+j} c^{j} d^{i+1-j} \\
& +\sum_{i=0}^{l}\left(\sum_{j=0}^{\min (i+1, l-i)}\left(\begin{array}{c}
i+1 \\
j
\end{array}\right)\left(\begin{array}{c}
l \\
i+j
\end{array}\right)\right) c^{l-i} d^{2 i+1} .
\end{aligned}
$$


The terms of lowest and highest degrees in the four sums are given by

$$
\begin{aligned}
& \mathrm{o}^{J}\left(z_{0}\right)=a^{n} c+c^{l+1}+\ldots+b^{n} c d^{n}+c d^{2 l}, \\
& \mathrm{o}^{J}\left(z_{1}\right)=a^{n} d+n a^{n-1} b c+(l+1) c^{l} d+\ldots+b^{n} d^{n+1}+d^{2 l+1} .
\end{aligned}
$$

At least in the case $l=n$ we get a regular sequence. However, before calculating a single obstruction we have $4 \geqslant \operatorname{dim}_{\text {Krull }} H \geqslant 2$ while the standard estimate yields $4 \geqslant \operatorname{dim}_{\text {Krull }} H \geqslant 0$. If we localise $B$ (and $M$ ) at the maximal ideal $\mathfrak{m}=(x, y)$, then

there is a natural isomorphism $\operatorname{Def}_{M}^{B} \rightarrow \operatorname{Def}_{\Omega_{B}(M)}^{B}$ given by mapping a deformation

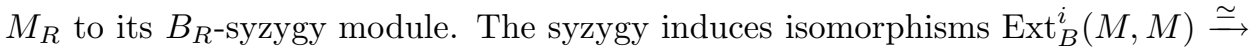
$\operatorname{Ext}_{B}^{i}\left(\Omega_{B}(M), \Omega_{B}(M)\right)$ for $i=1,2$; see [8]. In [9] we more generally show that the hull of the deformation functor of a rank 1 maximal Cohen Macaulay module $N$ on a hypersurface singularity Spec $B$ in particular satisfies the sharpened estimate

$$
\operatorname{dim}_{k} \operatorname{Ext}_{B}^{1} \geqslant \operatorname{dim}_{\text {Krull }} H \geqslant \operatorname{dim}_{k} \operatorname{Ext}_{B}^{1}-\operatorname{dim}_{k} \operatorname{Ext}_{B}^{2}+\operatorname{dim}_{k} \mathrm{H}_{2}(\mathcal{S}),
$$

where $\operatorname{Ext}_{B}^{i}=\operatorname{Ext}_{B}^{i}(N, N)$ and $\mathcal{S}=\mathcal{S}(\varphi)$ is the "Scandinavian complex" of the (square) presenting matrix $\varphi$ of $N$. In our case $\mathrm{H}_{2}(\mathcal{S}) \cong M \cong k^{2}$ (k-linearly).

The versal family of $\operatorname{Def}_{M}^{B}$ is the cyclic module $A \hat{\otimes} H /\left(y-a-x b, x^{2}-c-x d\right)$ where $A \hat{\otimes} H=\lim \left\{A \otimes H / \operatorname{im}\left(\mathfrak{m}_{H}^{n}\right)\right\}$. To find the obstruction one deforms the "generalised matrix factorisation" (see [8, Def. 6.1.6]) $\left(\left(y, x^{2}\right),\left(y^{n}, x^{m-1}\right)^{\mathrm{t}}\right)$ of $f=$ $x^{m+1}+y^{n+1}$. The universal lifting $M_{1}$ of $M$ to the Zariski tangent space $\operatorname{Def}_{M}^{B}(k[\varepsilon])$ is given by

$$
x^{m+1}+y^{n+1}=\left(y-a-x b, x^{2}-c-x d\right) \cdot\left(\begin{array}{c}
y^{n}+y^{n-1}(a+x b) \\
x^{m-1}+x^{m-3}(c+x d)
\end{array}\right) \quad \bmod \mathfrak{m}_{H}^{2} .
$$

To find the obstruction one has to lift and expand this factorisation as to be valid $\bmod \mathfrak{m}_{H}^{n}$ successively for all $n \geqslant 2$.

\section{ACKNowledgement}

The author is indebted to Professor O. A. Laudal for sharing essential insight and for valuable comments on the manuscript.

\section{REFERENCES}

[1] Michael Artin, Versal deformations and algebraic stacks, Invent. Math. 27 (1974), 165-189. MR 53:2945

[2] Inger Christin Borge, A cohomological approach to the modular isomorphism problem, Preprint in Pure Math. No. 15, Dept. of Math., University of Oslo, August 2002, www.math.uio.no/eprint/pure_math/2002/15-02, submitted to J. Pure Appl. Algebra.

[3] Inger Christin Borge and Olav Arnfinn Laudal, The modular isomorphism problem, Preprint in Pure Math. No. 25, Dep. of Math., University of Oslo, July 2003, www.math.uio.no/eprint/pure_math/2003/25-03, submitted to Invent. Math.

[4] David Eisenbud, Homological algebra on a complete intersection, with an application to group representations, Trans. Amer. Math. Soc. 260 (1980), no. 1, 35-64. MR 82d:13013

[5] Renée Elkik, Solutions d'équations a coefficients dans un anneau hensélien, Ann. Sci. École Norm. Sup. (4) 6 (1973), 553-604. MR 49:10692

[6] Barbara Fantechi and Marco Manetti, Obstruction calculus for functors of Artin rings, I, J. Algebra 202 (1998), 541-576. MR 99f:14004

[7] Gunnar Fløystad, Determining obstructions for space curves, with applications to nonreduced components of the Hilbert scheme, J. Reine Angew. Math. 439 (1993), 11-44. MR 94e:14004

[8] Runar Ile, Obstructions to deforming modules, Ph.D. thesis, University of Oslo, 2001. 
[9] _ Deformation theory of rank 1 maximal Cohen-Macaulay modules on hypersurface singularities and the Scandinavian complex, 2002, To appear in Compositio Math.

[10] Luc Illusie, Complexe cotangent et déformations I, Lecture Notes in Math., no. 239, SpringerVerlag, 1971. MR 58:10886a

[11] Complexe cotangent et déformations II, Lecture Notes in Math., no. 283, SpringerVerlag, 1972. MR 58:10886b

[12] Akira Ishii, Versal deformation of reflexive modules over rational double points, Math. Ann. 317 (2000), 239-262. MR 2001i:14005

[13] Yujiro Kawamata, Unobstructed deformations. II, J. Algebraic Geom. 4 (1995), 277-279. MR 96a:14014

[14] Olav Arnfinn Laudal, Formal moduli of algebraic structures, Lecture Notes in Math., no. 754, Springer-Verlag, 1979. MR 82h:14009

[15] - Matric Massey products and formal moduli I, Algebra, Algebraic Topology and Their Interactions, Lecture Notes in Math., no. 683, Springer-Verlag, 1986, pp. 218-240. MR 87k:55023

[16] Barry Mazur, Deforming Galois representations, Galois Groups over $\mathbb{Q}$ (Y. Ihara, K. Ribet, and J.-P. Serre, eds.), MSRI Publications, no. 16, Springer-Verlag, 1989, pp. 385-437. MR 90k:11057

[17] _ Deformation theory of Galois representations, Modular Forms and Fermat's Last Theorem (Gary Cornell, Joseph H. Silvermann, and Glenn Stevens, eds.), Springer-Verlag, 1997, pp. 243-311.

[18] Victor P. Palamodov, Deformations of complex spaces, Russian Math. Surveys 31 (1976), no. 3, 129-194. MR 58:22671

[19] Michael Schlessinger, Functors of Artin rings, Trans. Amer. Math. Soc. 130 (1968), 208-222. MR 36:184

[20] Arvid Siqveland, Global matric Massey products and the compactified Jacobian of the $\mathbf{E}_{\mathbf{6}}$ singularity, J. Algebra 241 (2001), 259-291. MR 2002d:14001

[21] _ The method of computing formal moduli, J. Algebra 241 (2001), 292-327. MR 2002g: 16050

[22] Hartwig von Essen, Nonflat deformations of modules and isolated singularities, Math. Ann. 287 (1990), no. 3, 413-427. MR 91j:14007

[23] Charles H. Walter, Some examples of obstructed curves in $\mathbb{P}^{3}$, Complex Projective Geometry (Trieste, 1989/Bergen, 1989), London Math. Soc. Lecture Note Ser., no. 179, Cambridge Univ. Press, 1992, pp. 324-340. MR 94a:14033

Department of Mathematics, University of Oslo, P.O. Box 1053 Blindern, NO-0316 OSLO, NORWAY

E-mail address: ile@math.uio.no 